\title{
Chapter 7 \\ Particle Identification: Time-of-Flight, Cherenkov and Transition Radiation Detectors
}

\author{
Roger Forty and Olav Ullaland
}

\subsection{Introduction}

Particle identification, PID, is of crucial importance in most experiments. The requirement can range from positive $\pi / \mathrm{K}$ identification in B-physics channels like $\mathrm{B}_{\mathrm{s}}^{0} \rightarrow \mathrm{D}_{\mathrm{s}}^{\mp} \mathrm{K}^{ \pm}$against a background from $\mathrm{B}_{\mathrm{s}}^{0} \rightarrow \mathrm{D}_{\mathrm{s}}^{-} \pi^{+}$which is $\sim 15$ times more abundant, to e/ $\pi$ separation at the level of $\sim 10^{-2}$ for momenta $>1 \mathrm{GeV} / c$ in order to effectively suppress a combinatorial background in channels like leptonic decays of heavy vector resonances.

The detectors should be non-destructive and should in addition introduce as little radiation length or interaction length as possible. We will in this chapter examine three experimental techniques which can be deployed for charged particle identification.

That is Time-of-Flight, Sect.7.2, and Cherenkov detectors which measure the particle velocity relative to the speed of light in vacuum, $\beta=v / c$, Sect. 7.4, and transition radiation detectors which are sensitive to $\gamma=1 / \sqrt{\left(1-\beta^{2}\right)}$ of the charged particle, Sect. 7.5. These detectors cannot be stand-alone detectors for PID purposes. They all require that the momentum of the particle is defined by other means, see Sect. 4.3, and then

$$
\frac{m^{2}}{p^{2}}=\frac{1}{\gamma^{2}-1}=\frac{1-\beta^{2}}{\beta^{2}}
$$

allowing the mass $m$ of the particle to be determined.

Only a limited amount of theory is included in this chapter as this is covered elsewhere in this book. The main emphasis will be on the working principles of these

R. Forty $(\bowtie) \cdot$ O. Ullaland CERN, Geneva, Switzerland e-mail: Roger.Forty@cern.ch 


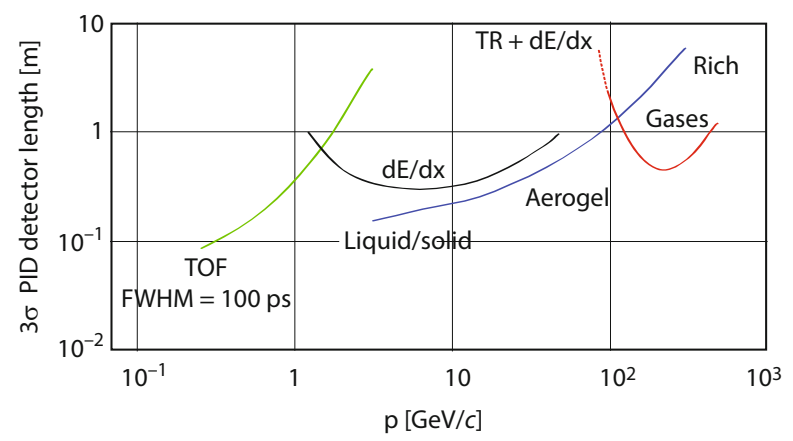

Fig. 7.1 Pion-kaon separation by different PID methods: the length of the detectors needed for 3 sigma separation. Adapted from [1]

detectors and how they are incorporated into compound experiments. A graphic representation of the different identification techniques can be seen in Fig. 7.1.

\subsection{Time of Flight Measurements}

The mass identification, $m_{i}$, of a momentum defined, $p_{i}$, charged particle is straight forward by measuring the flight time, $t_{i}$, over a path length, $l$. The mass, momentum, path length and flight time are related by:

$$
m_{i}^{2}=\frac{p_{i}^{2}}{l^{2}}\left[c t_{i}-l\right]\left[c t_{i}+l\right]
$$

and the uncertainties by:

$$
\left(\frac{\Delta m}{m}\right)^{2}=\left(\frac{\Delta p}{p}\right)^{2}+\gamma^{4}\left[\left(\frac{\Delta t}{t}\right)^{2}+\left(\frac{\Delta l}{l}\right)^{2}\right]
$$

There are essentially two sources of errors ${ }^{1}$ in the measurement of time, $t$, in Eq. (7.3).

1. The limitation of the electronics to resolve short time intervals. A random time jitter in the pulse height at the detector and thereby a time slewing or time walk.

2. Variation of the transit time of the photons or the free electrons, and thereby the signal formation time, in the detector.

\footnotetext{
${ }^{1}$ Irresolution was proposed in [2]. Although a nice word, it did not catch on.
} 


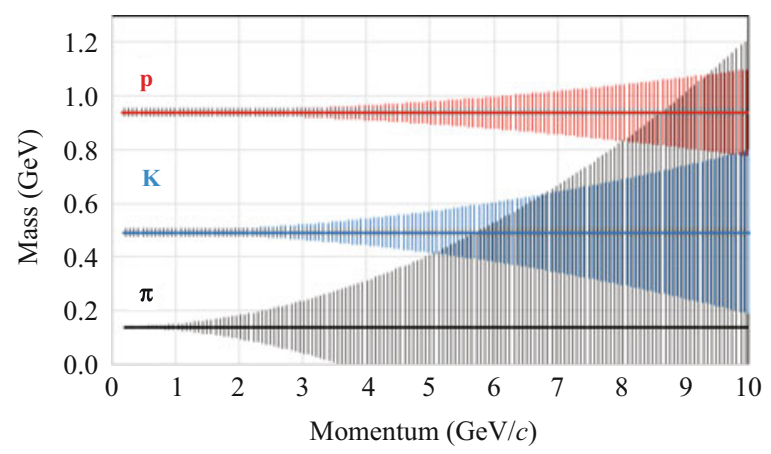

Fig. 7.2 Mass resolution as function of momentum for a Time of Flight, ToF, detector with $\Delta p / p=4 \cdot 10^{-3}, l=10 \mathrm{~m}, \Delta l / l=10^{-4}$ and $\Delta t=50 \mathrm{ps}$

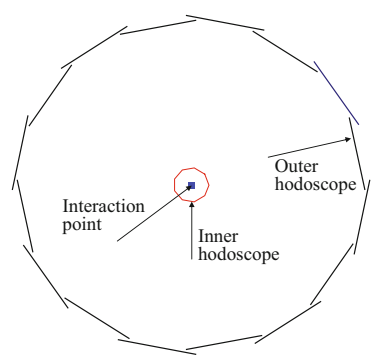

(a)

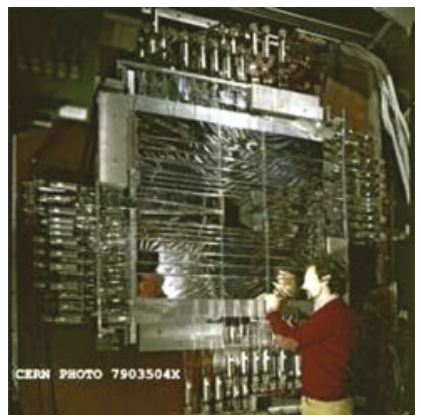

(b)

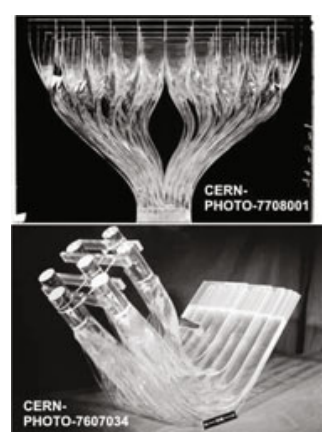

(c)

Fig. 7.3 (a) Simplistic sketch of a Time of Flight system. (b) Large scintillator hodoscope from CERN experiment NA1. (c) Light guides and scintillators

Particle misidentification will therefore occur when the time difference between two particles with the same momentum becomes comparable to the detector resolution. Figure 7.2 gives the mass resolution as function of momentum for $\pi$, $\mathrm{K}$ and proton.

Time of Flight detectors, ToF, have throughout been essential tools in physics experiments and have undergone impressive improvements in time resolution from micro-seconds to pico-seconds. The basis was worked out in [3]. A principle sketch is given in Fig. 7.3a in the Centre of Mass coordinate system. The interaction point is surrounded by a time zero hodoscope, the Inner hodoscope. Another hodoscope, the Outer hodoscope, is placed at a distance $l$ from the first one. Assuming that there is a momentum measurement between the two, this is all that is needed to solve Eq. (7.2).

The Inner hodoscope is usually not required. In a colliding beam experiment, the $\mathrm{RF}$ structure can be adequate to give a sufficiently precise time zero. In events with 
a large number of secondaries, one can use the feature that at least one particle will have a velocity $v \cong c$ and thereby use this one to define time zero.

The main work during the last years [4] has been in the improvement of the time resolution and, as the detectors have gradually increased in size, in the cost $/ \mathrm{m}^{2}$. The occupancy and radiation tolerance are playing a very important role for detectors that are proposed for the new high luminosity accelerators. We will here not explain the working principle of the detectors themselves. The reader is referred to Chap. 3. We will rather discuss the advantages and inconveniences of some of the most commonly used detector set-ups.

\subsubsection{Scintillator Hodoscopes}

A scintillator, read out in both ends by a photomultiplier, is the classic element of a Time of Flight hodoscope, Fig. 7.3b. The number of photons created is large. Plastic scintillators, as discussed in Chap. 3, have a density $\rho \simeq 1.03 \mathrm{~g} / \mathrm{cm}^{3}$. About $10^{4}$ photons $/ \mathrm{MeV}$ are created with a mean wavelength of $\sim 400 \mathrm{~nm}$ and a time constant $\tau \sim 1.5 \mathrm{~ns}$. The number of emitted photons per time unit, $\mathrm{N}$, will be approximately:

$$
N=\frac{N_{0}}{\tau} \exp \left(-\frac{t}{\tau}\right)
$$

$N$ is the number of photons emitted at time $t, N_{0}$ is the total number of emitted photons and $\tau$ is the average lifetime. $\tau$ is characteristic to a specific scintillator material. A short decay time increases the maximum count rate and is therefore an important property for detection. Most inorganic scintillators have rather long decay times, $\tau \sim 100 \mathrm{~ns}$, but in some cases the decay constant can be very short. For example, $\tau=1 \mathrm{~ns}$ for $\mathrm{BaF}_{2}$.

The specific energy loss, Chap. 2, for a minimum ionizing particle, MIP, is given as:

$$
\left(-\frac{d E}{d x}\right)_{\text {min }}=2.35-1.47 \ln (Z) \quad \mathrm{MeVcm}^{2} / \mathrm{g}
$$

where $Z$ is the atomic number.

$(d E / d x)_{\min }$ for a plastic scintillator is about $2 \mathrm{MeV} \mathrm{cm}^{2} / \mathrm{g}$, or about $2 \cdot 10^{4}$ photons $/ \mathrm{cm}$ are produced. This number of detectable photons will be greatly reduced due to the attenuation length of the material, the losses out from the material, quantum efficiency of the photon detector and the shaping time of the electronics. As the final number of photoelectrons is heavily dependent on the exact lay-out of the detector, it is very difficult to give a typical number. But, as a rule of thumb, approximately $2 \cdot 10^{-3}$ photoelectrons will be produced by the primary photon. This would give in the range of 40 photoelectrons $/ \mathrm{cm}$ in a plastic 
scintillator. Let $N_{\mathrm{D}}$ be the total number of detected photons. The time resolution is roughly proportional to $1 / \sqrt{N_{\mathrm{D}}}$. ToF detectors with high resolution, $\Delta t \sim 100 \mathrm{ps}$, therefore use scintillator thickness of $2-3 \mathrm{~cm}$. The material budget then becomes important.

The connection between the scintillator and the photon detector is a very important step in order to maximise the light collection efficiency of the system. These light concentrators are normally built around a Winston Cone [5] or a fishtail as in Fig. 7.3b. A Winston Cone is a non-imaging off-axis parabola of revolution which will maximise the collection of incoming rays. The ideal concentrator will achieve the highest possible concentration of radiant energy permitted by the second law of thermodynamics. This is equivalent to the general theorem of Liouville [6]. More specific in a case of a light guide, one can write:

$$
n^{2}-1 \geq\left[\frac{d}{2 r}+1\right]^{2}
$$

where $d$ is the light guide diameter and $r$ is the bending radius. $n$ is the refractive index relative to air. See Fig. 7.3c. Charged particles going through the light guides will give signal due to Cherenkov radiation and thereby give rise to an event correlated background.

A well designed scintillator for ToF must provide a good photon collection efficiency and a small time jitter. For fast timing one would normally rely on the first direct photon impact. This puts further constrains on the photon detector. The classic photon detector is the photomultiplier tube (PMT). Depending on the window geometry, dynode chain and HV configuration, the transient time spread is in the range of $1 \mathrm{~ns}$. This can be reduced by instrumenting both ends of the scintillator and then use mean timing. This will also take care of the after-pulsing in the PMT. These are normally either due to ions in the residual gas in the PMT which drift back, strike the photo cathode and liberate new photoelectrons or light from the dynodes which hit the photo cathode. The first will give a signal about $100 \mathrm{~ns}$ after the event, while the latter signal comes after 30-60 ns. See Chap. 3 for more information. However, still to overcome the path length and transient time variation, the detector has to output a large amount of primary photons to achieve total time resolution in the range of $100 \mathrm{ps}$.

An example can be found in [7]. Mean timing and time slewing corrections are performed. Slew-correction time, $T^{\text {cor }}$, is defined as:

$$
T^{\mathrm{cor}}=T+\frac{A_{0}}{\sqrt{\mathrm{ADC}}}
$$

where the constant $A_{0}$ is normally evaluated for each PMT and ADC is the signal pulse height. They report a nearly constant time resolution of $\sigma \sim 55 \mathrm{ps}$ across a detector length of $15 \mathrm{~cm}$. 


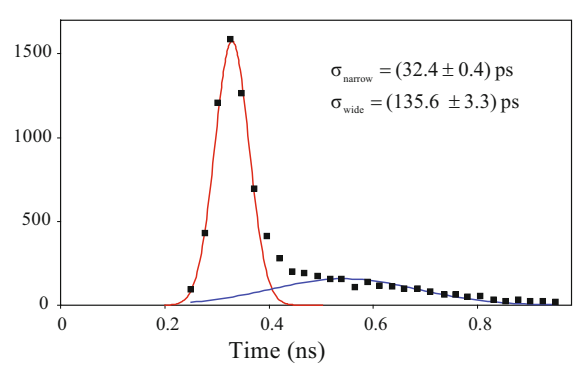

(a)

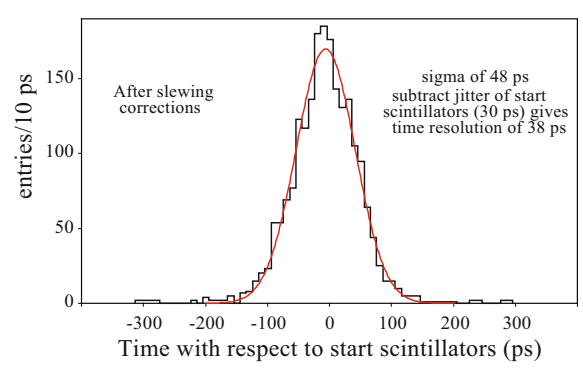

(b)

Fig. 7.4 (a) Single photoelectron timing resolution in Burle 64-pixel MCP-PMT 85012-501 with $10 \mu \mathrm{m}$ hole diameter. Adapted from [10]. (b) Time distribution of MRPC after slewing corrections. Adapted from [11]

Other photon detectors are generally faster and with smaller time spread than the PMT. See Chap. 3 for a detailed description of these devices. Below are some listed from [8]:

- $100 \mu \mathrm{m}$ diameter GaP SiPMT Avalanche Photo Diode operating in a Geiger mode with active quenching [9]. Single photoelectron regime: $25 \mathrm{ps}$

- Hamamatsu H-8500 Flat panel multi anode photo multiplier tube (MaPMT). ${ }^{2}$ SLAC measurement [8] of single photon resolution: $140 \mathrm{ps}$

- Burle 85011 photo multiplier tube with micro channel plate (MCP-PMT). ${ }^{3}$ SLAC measurement [8] of single photon resolution: $<50 \mathrm{ps}$

A drawback with these detectors can be the non-Gaussian tails as shown on Fig. 7.4a.

\subsubsection{Parallel Plate ToF Detectors}

One of the main challenges in using gas based detectors, MWPC up to spark chambers as discussed in Chap. 3, is the time jitter caused by the spread in pulse heights due to the long Landau tail. This can to some extent be overcome by using many gaps and operating the detector in a regime where the pulse height is nearly independent of the primary ionisation. However, this can seriously diminish the rate capability of these detectors. Well adapted electronics will furthermore decrease the time walk.

\footnotetext{
${ }^{2}$ HAMAMATSU PHOTONICS K.K. 325-6, Sunayama-cho, Naka-ku, Hamamatsu City, Shizuoka Pref., 430-8587, Japan.

${ }^{3}$ BURLE INDUSTRIES, INC. 1000 New Holland Avenue, Lancaster, PA 17601-5688 U.S.A.
} 

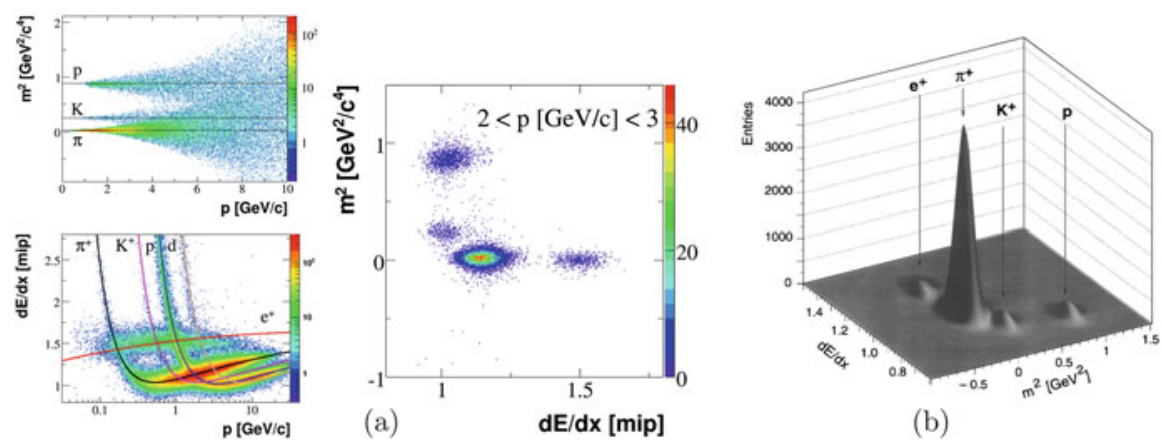

Fig. 7.5 (a) Particle identification in NA61. Reference [13]. (b) Particle identification at NA 49 by simultaneous $\mathrm{dE} / \mathrm{dx}$ and TOF measurement in the momentum range 5 to $6 \mathrm{GeV} / c$ for central $\mathrm{Pb}+\mathrm{Pb}$ collisions. Reference [14]

Large area resistive plate chambers, see Chap. 3, are successfully used as time of flight detectors. An example is the $\sim 150 \mathrm{~m}^{2}$, with $1.6 \cdot 10^{5}$ read-out channels, detector for ALICE [11]. Ten gaps of $250 \mu \mathrm{m}$ width are made from $400 \mu \mathrm{m}$ thick soda-lime glass with a gas composition of $\mathrm{C}_{2} \mathrm{H}_{2} \mathrm{~F}_{4}: \mathrm{SF}_{6}: \mathrm{C}_{4} \mathrm{H}_{10}=0.90: 0.05: 0.05$. The resistivity ${ }^{4}$ of the glass is $\sim 10^{13} \Omega \mathrm{cm}$. The detector is operated just below streamer mode. Tests indicate no change in performance up to $1 \mathrm{kHz} / \mathrm{cm}^{2}$. As there are many gaps, the output charge distribution is a broad, but nearly Gaussian distributed with some Landau tail towards higher value. This will give rise to some time slewing. The time resolution is given as $\sigma<40$ ps. See Fig. 7.4b.

\subsection{The Power of Combined PID}

The inherently simple ToF technique has greatly evolved over the years. The coming of the higher energy and/or higher intensity accelerators have required an ever better time and space resolution. Even though there has been great progress with small single pixel devices, progress with large systems has been slow. An overview of the current state of the art can be found in [12].

Combining different PID techniques, even with modest resolution, has been the preferred option for many experiments. An example of this powerful approach is shown in Fig. 7.5.

\footnotetext{
${ }^{4}$ It can be worth noting that materials which exhibit very large resistivity, might not be Ohmic, but rather ionic, and thereby show large variations depending on the applied current or voltage.
} 


\subsection{Cherenkov Radiation}

The theory of Cherenkov radiation is discussed in Chap. 2. Further reading can be found in references [15-18]. We will here just recall some of the main features. The condition for emission of a Cherenkov photon is given by

$$
\cos \Theta_{\mathrm{C}}=\frac{1}{\beta \cdot \sqrt{\varepsilon(\lambda)}}=\frac{1}{\beta \cdot n(\lambda)}
$$

and the number of emitted photons by

$$
\frac{d^{2} N}{d L d \lambda}=2 \pi \alpha Z^{2} \frac{\sin ^{2} \Theta_{\mathrm{C}}}{\lambda^{2}},
$$

where $\Theta_{C}$ is the angle of the emitted photon relative to the particle trajectory, $\varepsilon$ is the dielectric constant as function of the photon wavelength $\lambda, L$ is the radiator length, $\alpha \sim 1 / 137$ is the fine structure constant, $\beta$ is the particle velocity relative to the speed of light in vacuum, $\beta=v / c=p c / E$, and $Z$ is the charge of the particle in units of electron charges. The refractive index, $n$, is given as $n^{2}=\varepsilon$. The relationship between the photon wavelength and its angular frequency, $\omega$, is given by $\lambda(\mathrm{nm}) \sim 1240 / \hbar \omega(\mathrm{eV})$. A representation of the Cherenkov radiation domain is given in Fig. 7.6a.

From the discussion in Chap. 2 and Eq. (7.8) it is clear that $\varepsilon$ has to be real and larger than 1 and that the speed of the charged particle must be larger than the phase velocity of the electromagnetic fields at the frequency $\omega$ in order to have emission of Cherenkov photons at that frequency.

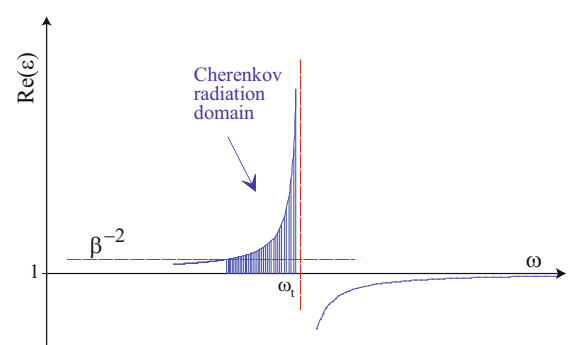

(a)

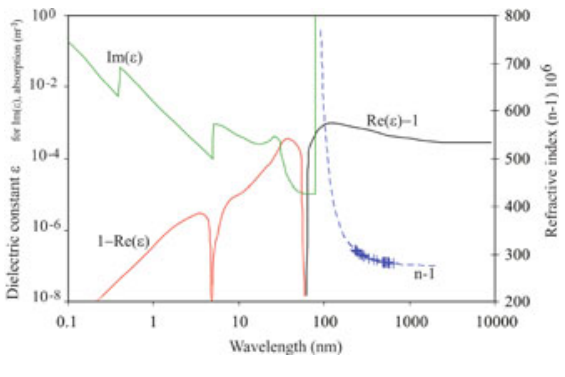

(b)

Fig. 7.6 (a) Simplistic representation of the real part of the dielectric constant, $\Re(\varepsilon)$, as function of the frequency, $\omega$. (b) The dielectric constant, $\varepsilon$, and the refractive index, $n$, for argon at $0{ }^{\circ} \mathrm{C}$ and $101.3 \mathrm{kPa} . \varepsilon$ data replotted from [19] and $n$ from [20] 
We see from the above that Cherenkov radiation is characterized by:

- Cherenkov radiation is a prompt signal.

- The existence of a threshold ${ }^{5}$ in $\beta_{\min }=n^{-1}$

- The Cherenkov angle is depending on $\beta$.

- The number of Cherenkov photons emitted is depending on $\beta$.

- The number of photons emitted is depending on the square of the charge of the particle.

The properties described above of Cherenkov radiation can be used to measure the velocity of a charged particle traversing matter. Consider two charged particles with known momenta $p$ and mass and velocity given by $m_{i}$ and $\beta_{i}$. The mass difference can then be written as:

$$
m_{1}^{2}-m_{2}^{2}=p^{2} \cdot \frac{\left(\beta_{1}-\beta_{2}\right)\left(\beta_{1}+\beta_{2}\right)}{\left(\beta_{1} \cdot \beta_{2}\right)^{2}}=n^{2} p^{2} \cdot\left(\cos ^{2} \Theta_{1}-\cos ^{2} \Theta_{2}\right)
$$

And if $n-1$ is small

$$
m_{1}^{2}-m_{2}^{2}=p^{2} \cdot\left(\Theta_{2}+\Theta_{1}\right)\left(\Theta_{2}-\Theta_{1}\right)
$$

The resolution in mass is thereby directly linked to the angular resolution of the detector. The main emphasis for all the Cherenkov detectors will be angular resolution.

The refractive index together with $\varepsilon$, for argon at $0^{\circ} \mathrm{C}$ and $101.3 \mathrm{kPa}$, is given in Fig. 7.6b. The data for the refractive index of argon is well described by a single pole Sellmeier, see Eq. (7.16), representation:

$$
(n-1) \cdot 10^{6}=\frac{0.05086}{73.82^{-2}-\lambda^{-2}}
$$

with $\lambda$ in $\mathrm{nm}$. We observe that this pole is where $\mathfrak{R}(\varepsilon)$ goes from larger than 1 to smaller than 1. At about the same wavelength $\Im(\varepsilon)$ becomes important.

A Cherenkov light detector is therefore based on classical optics. The choice of radiator, and thereby the refractive index, is depending on the momentum range which has to be covered and the photon detector option. We will in the following discuss different radiator materials, Sect.7.4.2, and the usage from Threshold, Sect. 7.4.3, to Ring Imaging Cherenkov detectors, Sect. 7.4.4. We will first take a closer look at the refractive index, Sect. 7.4.1.

\footnotetext{
${ }^{5}$ Due to diffraction broadening, Cherenkov photons can be emitted below threshold. We will not discuss that here.
} 
Table 7.1 Atomic refraction constants from Ref. [22]

\begin{tabular}{l|l|l}
\hline Atom & & Atomic refraction \\
\hline Carbon & & 2.418 \\
\hline Bromine & & 8.865 \\
\hline Chlorine & & 5.967 \\
\hline Fluorine & & 1.1 \\
\hline Hydrogen & & 1.1 \\
\hline Iodine & & 13.952 \\
\hline One double bond & $=\mathrm{O}$ & 2.122 \\
\hline Two single bonds & $-\mathrm{O}-$ & 1.643 \\
\hline
\end{tabular}

\subsubsection{Refractive Index}

The dielectric constant is given by:

$$
\varepsilon=1+4 \pi \chi=\frac{1+\frac{8}{3} \pi N \zeta}{1-\frac{4}{3} \pi N \zeta} \quad \text { from which } \quad \frac{4}{3} \pi N \zeta=\frac{\varepsilon-1}{\varepsilon+2}
$$

where $\chi$ is the susceptibility, $\mathrm{N}$ is the number of molecules per unit volume and $\zeta$ is the molecular polarizability.

A relation like this was first obtained by Mossotti in 1850, then by Lorenz in 1869 , and refined by Clausius in 1879 , and which is usually called the ClausiusMossotti equation. Polarizable matter was modelled as an assembly of small conducting spheres in the early studies. ${ }^{6}$ Maxwell's theory showed that the index of refraction of light, $n$, was related to $\varepsilon$ by $n^{2}=\varepsilon$, so that the formula could be applied to light as well as to static fields. H.A. Lorentz, in 1878, and L.V. Lorenz (1829-1891), in 1881, derived a similar formula on the basis of the electron theory in which $n^{2}$ replaced $\varepsilon$. This formula is called the Lorentz-Lorenz formula, and can be written in the following way:

$$
n^{2}=\frac{1+2\left(\rho \frac{R_{M}}{M_{W}}\right)}{1-\left(\rho \frac{R_{M}}{M_{W}}\right)}
$$

where $R_{M}$ is the molar refraction, $M_{W}$ is the molecular weight and $\rho$ is the density.

The molar refraction may then be estimated from the chemical formula. Atomic refraction constants differ slightly in the literature, but the constants in Table 7.1 give reasonable results for many compounds.

The Lorentz-Lorenz equation, Eq. (7.14) together with Table 7.1, does not explicitly express the refractive index as a function of the photon energy. ${ }^{6}$ Strictly speaking, Clausius-Mossotti equation is only rigorously valid in the limit of zero
density [21]. 
The most common way to represent the refractive index is in the form of a series with multiple poles

$$
n-1=C \cdot \sum_{i} \frac{f_{i}}{v_{i}^{2}-v^{2}} \quad \text { with } \quad C=\frac{e^{2} A}{2 \pi m c^{2}}=1.2098 \cdot 10^{6}
$$

where $e$ and $m$ are the charge and mass of the electron, $A$ is Avogadro's number per $\mathrm{cm}^{3}$ and $\nu\left(\mathrm{cm}^{-1}\right)=10^{7} / \lambda(\mathrm{nm}) . f_{i}$ is the oscillator strength of the Eigen frequency $\nu$. We will here mainly use the standard Sellmeier formula with one pole:

$$
\frac{3}{2} \cdot \frac{n^{2}-1}{n^{2}+2}=\frac{a}{\lambda_{0}^{-2}-\lambda^{-2}} \simeq n-1 \text { for } n-1 \ll 1
$$

$b=\lambda_{0}^{-2}$ will also be used. $\lambda$ is in nm. $a / b$ is the asymptotic value of $n$ as $\lambda \rightarrow \infty$. A two pole Sellmeier representation might be required:

$$
\frac{3}{2} \cdot \frac{n^{2}-1}{n^{2}+2}=\left[a_{1} \cdot \lambda^{-4}+a_{2} \cdot \lambda^{-2}+a_{3}\right]^{-1}
$$

Clearly also other types of power series can be used to approximate the refractive index like in reference [23]. In this case the refractive index is approximated with the half empirical formula of a n-term Cauchy equation which is very similar to Eq. (7.17):

$$
n-1=2 \pi N_{0}\left[a_{0}+a_{1} \omega^{2}+a_{2} \omega^{4}+a_{3} \omega^{6}\right]
$$

where $\omega$ is the frequency in atomic units. The $a_{3} \omega^{6}$ term has been added after the original series [24] was truncated at $a_{2} \omega^{4}$ and thereby was not very useful in the UV to VUV wavelength region.

The refractive index of a medium $M$ which is a mixture of different molecules in the ratio $M=\sum_{i}\left[M_{i} / f_{i}\right]$ for $1=\sum_{i} f_{i}^{-1}$, is given by $n_{M}=\sum_{i}\left[n_{i} / f_{i}\right]$. We will illustrate this with a simple example. The refractive index of air and its constituents are well measured quantities, Fig. 7.7a.

The Sellmeier parameterisation for $\mathrm{N}_{2}, \mathrm{O}_{2}, \mathrm{CO}_{2}$ and argon is given in Table 7.2. Note that whereas a single pole, Eq. (7.16), describes well $\mathrm{N}_{2}, \mathrm{CO}_{2}$ and argon, the data for $\mathrm{O}_{2}$ is best described with a two pole, Eq. (7.17), representation. The parameters used to describe the data points for dry air in Fig. $7.7 \mathrm{a}$ are

$$
\begin{aligned}
n(\text { air }) & =0.7809 \cdot n\left(\mathrm{~N}_{2}\right)+0.2095 \cdot n\left(\mathrm{O}_{2}\right)+0.0093 \cdot n(\mathrm{Ar})+0.0003 \cdot n\left(\mathrm{CO}_{2}\right) \\
& \simeq 1+10^{-6} \cdot\left[\left(\lambda^{-2}-69.1^{-2}\right)\left(\lambda^{-2}+99.5^{-2}\right)\right]^{-1}
\end{aligned}
$$




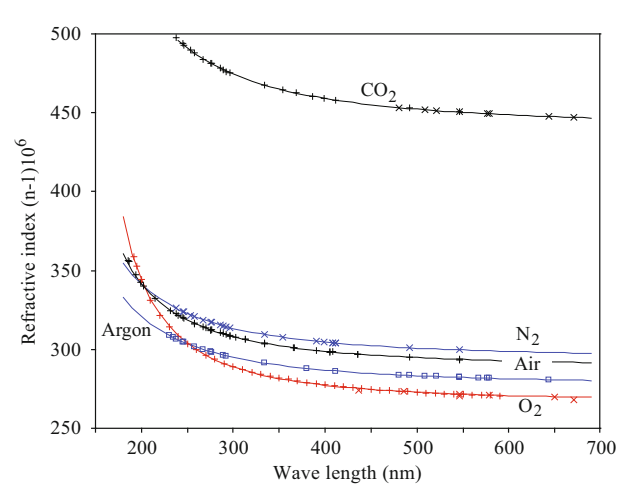

(a)

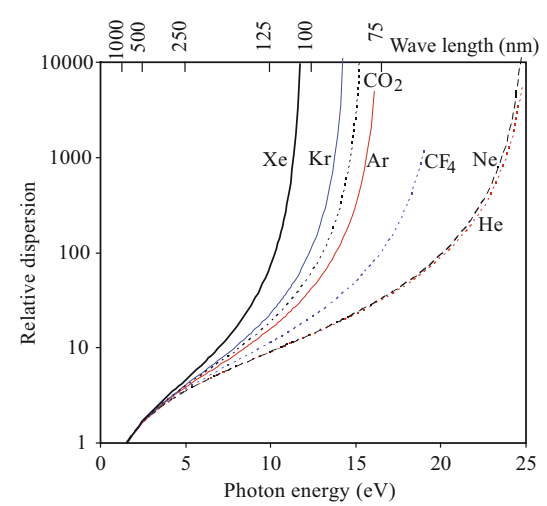

(b)

Fig. 7.7 (a) The refractive index of dry air, $\mathrm{N}_{2}, \mathrm{O}_{2}, \mathrm{CO}_{2}$ and argon at $0{ }^{\circ} \mathrm{C}$ and $101.3 \mathrm{kPa}$ [20]. (b) Dispersion $d n / d E$ relative to the value at $800 \mathrm{~nm}$, in some noble and n-atomic gases as function of the photon energy [20]

Table 7.2 Sellmeier fit, Eqs. (7.16) and (7.17), parameters for the gases at $0^{\circ} \mathrm{C}$ and $101.3 \mathrm{kPa}$

\begin{tabular}{l|l|l|l|l|l|r}
\hline $\mathrm{Gas}$ & $\mathrm{A}$ & $\mathrm{B}$ & $\mathrm{a}_{1}$ & $\mathrm{a}_{2}$ & $\mathrm{a}_{3}$ & $\lambda_{0}$ \\
\hline $\mathrm{N}_{2}$ & 0.0532 & 0.000181 & & & & 74.36 \\
\hline $\mathrm{O}_{2}$ & & & $-54,955$ & -20.275 & 0.00376 & 122.90 \\
\hline $\mathrm{CO}_{2}$ & 0.0687 & 0.000156 & & & & 80.10 \\
\hline $\mathrm{Ar}$ & 0.0509 & 0.000184 & & & & 73.82 \\
\hline
\end{tabular}

The pole, $\lambda_{0}$, in $\mathrm{nm}$. $\mathrm{O}_{2}$ has only one real pole in this representation

Although the last expression gives a good description of the refractive index for dry air at $0^{\circ} \mathrm{C}, 101.3 \mathrm{kPa}$ and for $\lambda \geq 130 \mathrm{~nm}$, the real pole at $\sim 69 \mathrm{~nm}$ has no physical meaning.

\subsubsection{Cherenkov Radiators}

Cherenkov radiators have to be reasonably optically transparent and with an appropriate refractive index. The scintillation and phosphorescence processes in the medium should be small. There is a wide variety to chose from, from transparent solids via liquids to gases. One can in addition change the refractive index by changing temperature and pressure of the medium.

The dispersion in a radiator can be written as

$$
\frac{d n}{d E} \propto \frac{\left(n^{2}-1\right)^{2}}{n} \cdot E \quad \text { and for }(n-1) \ll 1 \text { it reduces to } \quad \frac{d n}{d E} \propto(n-1)^{2} \cdot E
$$

where $E$ is the energy of the photon. 


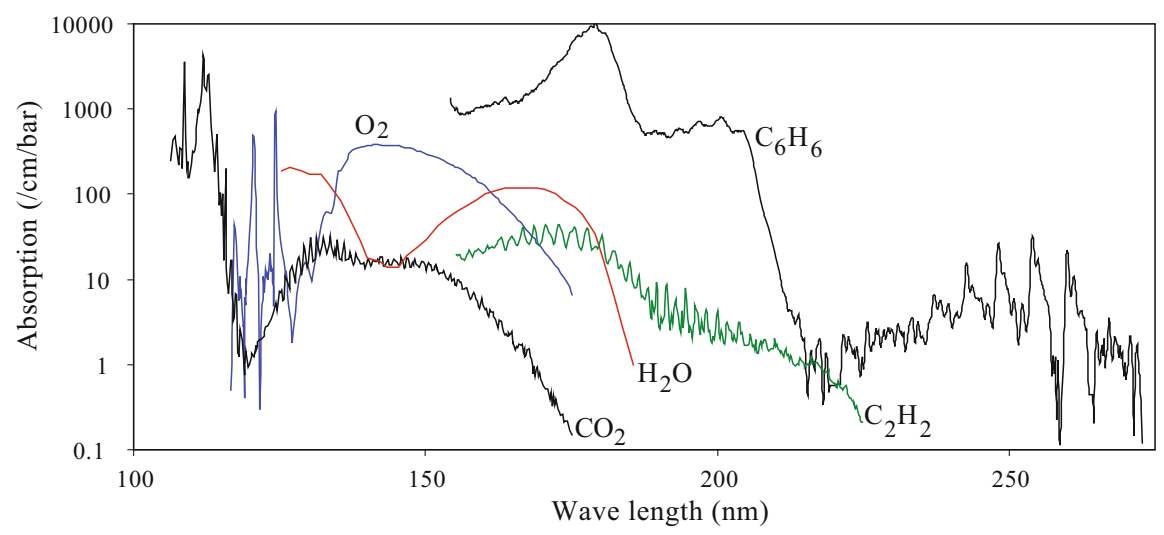

Fig. 7.8 Absorption as function of the photon wavelength [25]

The dispersion in some noble and n-atomic gases is plotted in Fig. 7.7b. He and $\mathrm{Ne}$ are very weakly dispersive in contrast to $\mathrm{Kr}$ and $\mathrm{Xe}$. As can be seen from Fig. 7.7b, fluorocarbons are also weakly dispersive. If the definition of the Cherenkov angle is an important quantity for the detector, it is then clear that the dispersion has to be as small as possible over the photon detector efficiency window. The detector design will be a balance between number of photons and the spread in the Cherenkov angle.

The radiator medium becomes opaque when the imaginary part of the dielectric constant becomes important, Fig. 7.6b. Most media will in addition exhibit broad and strong absorption bands. Figure 7.8 shows the absorption in some commonly used Cherenkov media or trace impurities in them. For simple alkanes, $\mathrm{C}_{\mathrm{N}} \mathrm{H}_{2+2 \mathrm{~N}}$, the onset of photon absorption [25] can be approximated to:

$$
\lambda_{\mathrm{CH}}(\mathrm{nm})=181-\frac{226}{3(\mathrm{~N}+1)}
$$

A similar approximation can be given for n-perfluorocarbons, $\mathrm{C}_{\mathrm{N}} \mathrm{F}_{2+2 \mathrm{~N}}$,

$$
\lambda_{\mathrm{CF}}(\mathrm{nm})=175.6-\frac{641}{3 \mathrm{~N}+5.7}
$$

It can be seen from these two expressions that n-perfluorocarbons are more transparent than alkanes. Alkanes are therefore good quenchers as used in MWPCs. Trace impurities are particularly difficult to eliminate especially when it is not clear which molecule is causing the absorption. The successful detector design should therefore not be sensitive to these bands. 


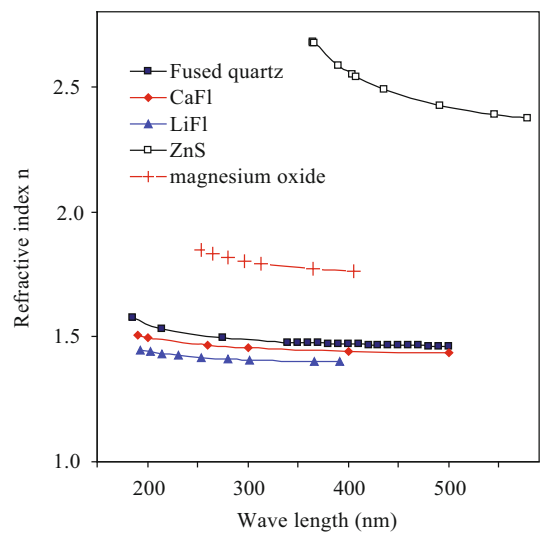

(a)

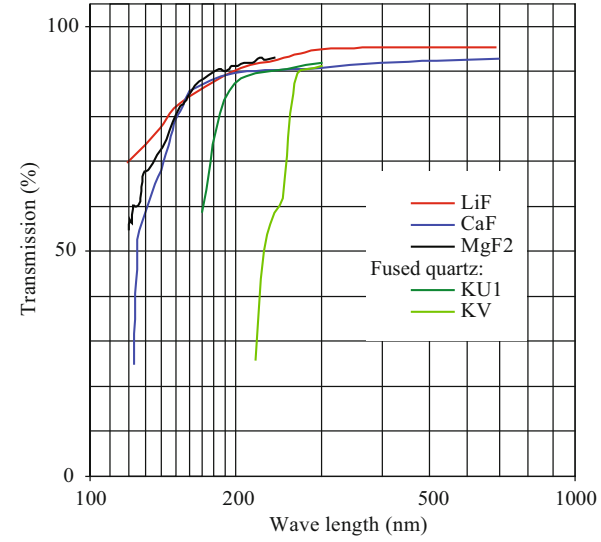

(b)

Fig. 7.9 (a) Refractive index for quartz and other special optical materials [26]. (b) Transmission in some commercially available quartz as function of wavelength. See footnote 7

\subsubsection{Quartz Radiators}

Quartz radiators are very popular for Cherenkov detectors operating in the low momentum range. The refractive index for some quartz and other optical materials is given in Fig. 7.9a. Figure 7.9b gives the transmission for some commercially ${ }^{7}$ available quartz. By choosing a refractive index $n \sim 1.5$ and a photon detection window from 800 to $300 \mathrm{~nm}$, the Cherenkov angle measurement between $\pi$ and $\mathrm{K}$ becomes difficult for $p>2 \mathrm{GeV} / c$ due to dispersion.

Quartz radiators in Cherenkov detectors are treated in two distinctly different ways. We see that for the $n \sim 1.5$ quartz, a $\pi$ will pass the Cherenkov threshold at $125 \mathrm{MeV} / c$ and at $\sim 280 \mathrm{MeV} / c$ no light will escape the quartz due to total internal reflection for particles perpendicular onto the radiator. An elegant solution to this problem is shown in Fig. 7.10a.

The other option is to exploit the feature of internal reflections as a light guide for the Cherenkov photons. The working principle of a DIRC, Detection (of) Internally Reflected Cherenkov (light) [28], detector is sketched in Fig. 7.10b. The standoff region is designed to maximize the transfer efficiency between the radiator and the detector. If this region has the same index of refraction as the radiator, $n_{1} \simeq n_{2}$, the transfer efficiency is maximized and the image will emerge without reflection or refraction at the end surface. Further improvements can be achieved by measuring the transfer time of the Cherenkov photons [29]. A large fraction of the uncertainties caused by the dispersion can then be eliminated.

\footnotetext{
${ }^{7}$ Data from:

Del Mar Ventures, 12595 Ruette Alliante No.148, San Diego, California 92130, US.

Crystran Ltd, 1 Broom Road Business Park, Poole, Dorset, England.
} 


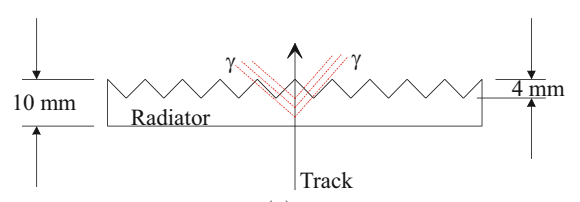

(a)

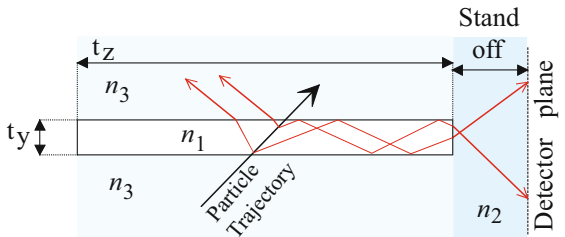

(b)

Fig. 7.10 (a) Sketch of the saw tooth quartz radiator for CLEO 3 [27]. (b) Schematic of the radiator bar for a DIRC [28] detector. Not to scale

Similar, but not identical, are the Time-of-Propagation, TOP [30] detector at the BELLE II experiment and the proposed detectors; TORCH [32] at LHCb and a DIRC [33] at the PANDA experiment.

The TOP consists of quartz radiator bars $270 \mathrm{~cm}$ long $\times 45 \mathrm{~cm}$ wide $\times 2 \mathrm{~cm}$ thick. See Fig. 7.11a. One end of the bar has a spherical mirror to reflect light back to the other end that has a small expansion prism. The prism is instrumented with 32 16-channel microchannel plate photomultiplier tubes (MCP-PMTs) readout with custom giga-sample per second waveform sampling electronics. The Cherenkov ring is imaged by the 512 MCP-PMT pixels with $5 \mathrm{~mm}$ pixel size and the time of arrival of each photon is measured with $<50 \mathrm{ps}$ timing resolution. The photon time of arrival is a sum of the time of flight of the charged particle to the quartz radiator and the time of propagation of the Cherenkov photons to the photodetectors. Results with test beam data is shown in Fig. 7.11b. Clear $\pi / \mathrm{K}$ separation can be observed. The detector will be ready for data taking in 2018 .

\subsubsection{Aerogel Radiators}

The search for a stable Cherenkov radiator with a refractive index between gas and liquid started about the same time as the first Cherenkov detector became operational. The first successful was silica aerogel [34]. The Axial Field Spectrometer [35] at the CERN ISR was the first large experiment to use it. The principle fabrication reactions ${ }^{8}$ are rather simple:

$$
\begin{aligned}
& \mathrm{Si}\left(\mathrm{OCH}_{3}\right)_{4}+4 \mathrm{H}_{2} \mathrm{O} \stackrel{\mathrm{NH}_{3}}{\longrightarrow} \mathrm{Si}(\mathrm{OH})_{4}+4 \mathrm{CH}_{3} \mathrm{OH} \\
& \mathrm{nSi}(\mathrm{OH})_{4} \longrightarrow\left(\mathrm{SiO}_{2}\right)_{\mathrm{n}}+2 \mathrm{nH}_{2} \mathrm{O}
\end{aligned}
$$

The refractive index, $n$, as a function of the wavelength, $\lambda$, can be approximated by $n=1+k \cdot \rho$ for the density $\rho$ in $\mathrm{g} / \mathrm{cm}^{3}$ and $k$ is a function of $\lambda$. An example

\footnotetext{
${ }^{8}$ Tetramethyl orthosilicate is used in this example. Tetraethyl orthosilicate can also be used and is normally preferred as the byproduct is ethanol rather than methanol. Both tetramethyl orthosilicate and tetraethyl orthosilicate are highly reactive [36].
} 

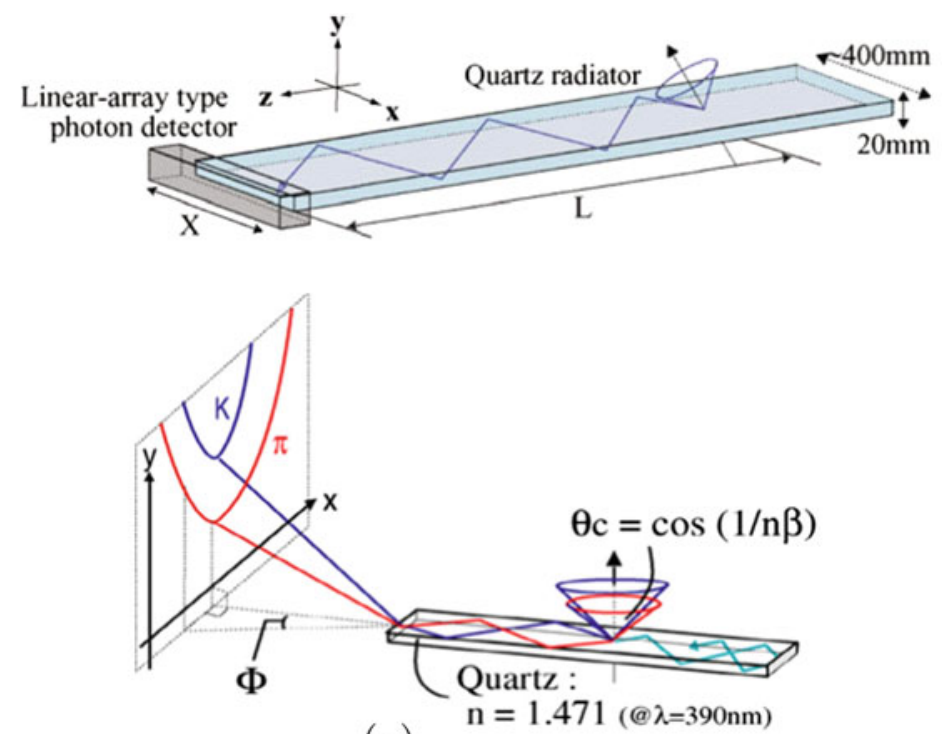

(a)
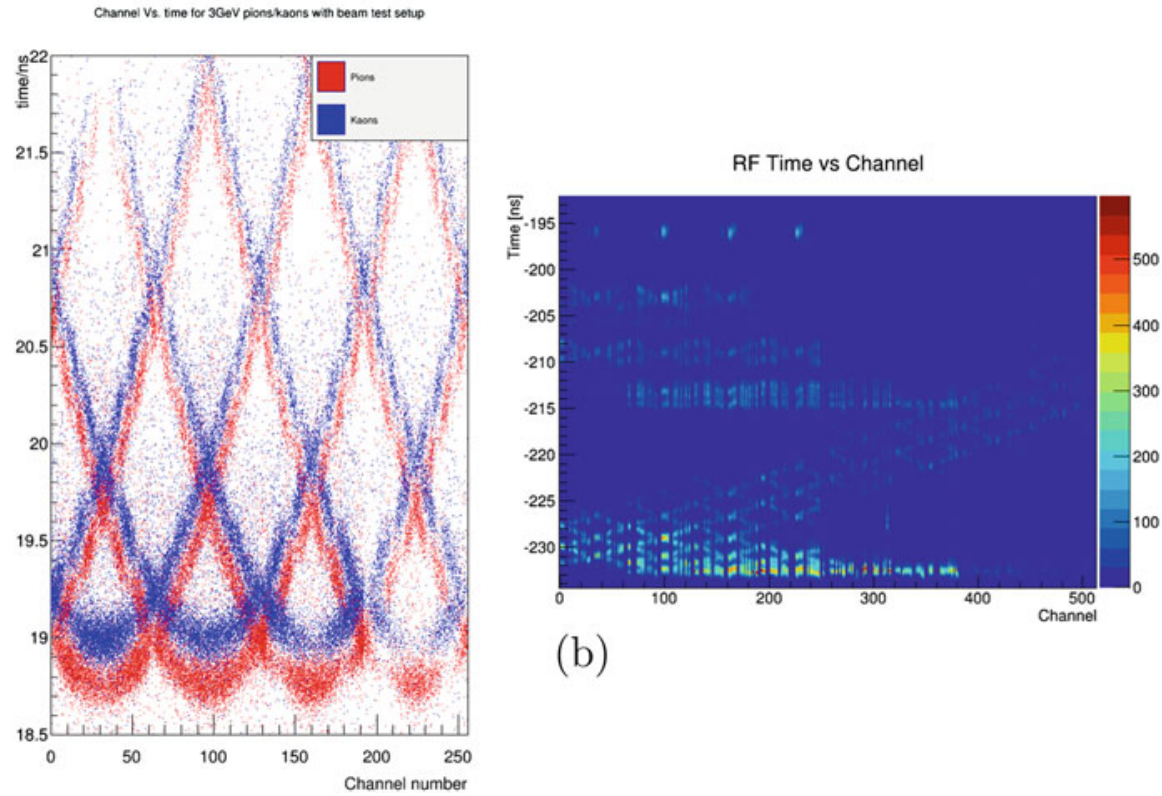

(b)

Fig. 7.11 (a) Schematic drawing of a TOP-counter. Reference [30]. (b) Test beam data. Cumulative distribution of measured time versus channel number for detected photons; the insert shows a zoom at short times, indicating the separation in time between the signals from pions and kaons. Reference [31] 


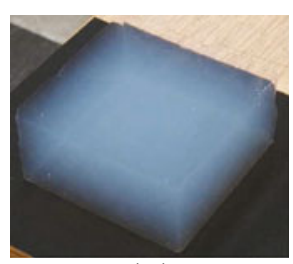

(a)

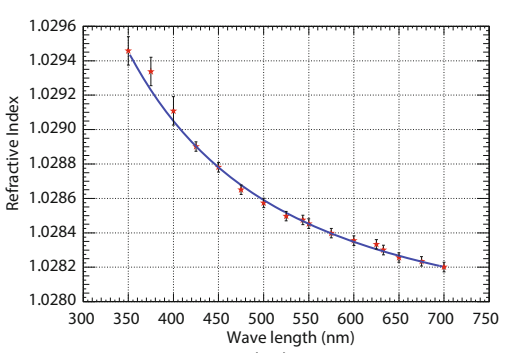

(b)

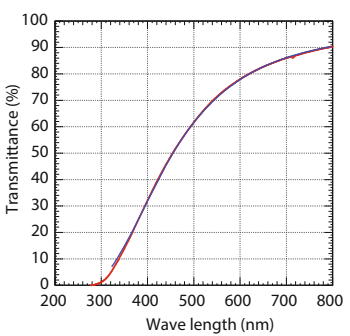

(c)

Fig. 7.12 (a) Aerogel tile. Courtesy of the LHCb Milano group. (b) Refractive index of aerogel as function of wavelength. Bellunato et al. [37] with permission. (c) Transmittance of $52.10 \mathrm{~mm}$ thick aerogel as function of wavelength. Perego [38] with permission

is shown in Fig. 7.12b. The data [37] is well described by a single pole Sellmeier equation:

$$
n^{2}-1=\frac{a_{0} \lambda^{2}}{\lambda^{2}-\lambda_{0}^{2}}
$$

for $a_{0}=0.05639 \pm 0.00004$ and $\lambda_{0}=(83.22 \pm 1.25) \mathrm{nm}$.

Assuming that aerogel is just a rarefied form of silica, $a_{0}$ and the density of the material are linked by:

$$
\rho(\text { aerogel })=\frac{a_{0}(\text { aerogel })}{a_{0}\left(\mathrm{SiO}_{2}\right)} \frac{n^{2}\left(\mathrm{SiO}_{2}\right)+2}{n^{2}(\text { aerogel })+2} \rho\left(\mathrm{SiO}_{2}\right)
$$

which gives $\rho$ (aerogel $)=(0.158 \pm 0.001) \mathrm{g} / \mathrm{cm}^{3}$, in reasonably good agreement with $\rho=(0.149 \pm 0.004) \mathrm{g} / \mathrm{cm}^{3}$ which was given by the manufacturer.

Two main types of aerogel are now available, hydrophobic ${ }^{9}$ and hygroscopic. ${ }^{10}$ Large homogeneous blocks of high optical quality are now readily available. The refractive index can be tuned between 1.008 and 1.1. By stacking aerogel blocks of different refractive indices, the total light output can be increased while minimizing the width of the Cherenkov ring. By modifying the reaction conditions of the sol-gel synthesis [39], it is possible to control the variations of $n$ inside the aerogel tile and thereby create a monolithic block with well defined different layers of $n$.

\footnotetext{
${ }^{9}$ Advanced Technology Research Laboratory, 1048 Kadoma, Kadoma-shi, Osaka-fu, Japan 571.

${ }^{10}$ Boreskov Institute for Catalysis in collaboration with the Budker Institute of Nuclear Physics in Novosibirsk.
} 


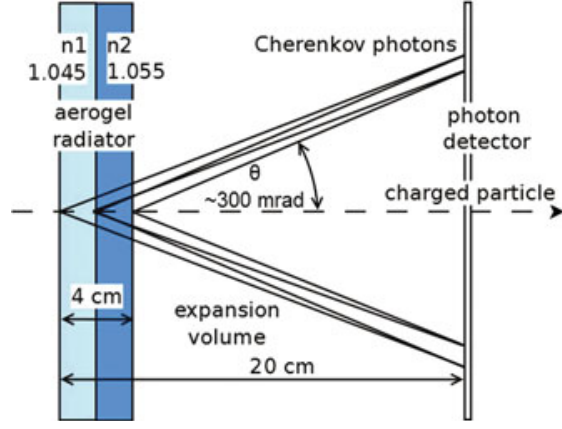

(a)

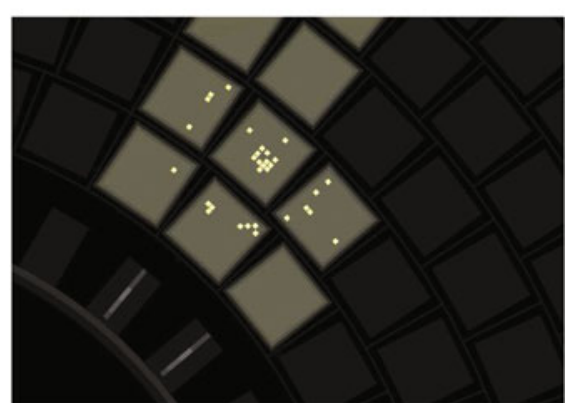

(b)

Fig. 7.13 (a) Proximity focusing RICH with two layers of the aerogel radiator: Cherenkov photons emitted in two aerogel tiles are detected on the same ring by the position sensitive photon detector, thus reducing the ring width. (b) Cosmic ray events registered by partially equipped detector. Reference [41]

The optical quality, light transmission $T$, see Fig. 7.12c, of aerogel is normally parameterized as:

$$
T=T_{0} \cdot \exp \left[-C \cdot \frac{t}{\lambda^{4}}\right]
$$

where $C$ is the clarity given in $\mu \mathrm{m}^{4} / \mathrm{cm}$ and $t$ is the thickness in $\mathrm{cm} . T_{0}$ describes the bulk properties of the aerogel and $C$ the variation with the wavelength. The $\lambda^{4}$ term shows that the light attenuation, opacity $\kappa,{ }^{11}$ is governed by Rayleigh scattering [40] which can be written as:

$$
\kappa=\sigma_{\mathrm{Rlh}}(\lambda) \cdot N_{0} \cdot t, \text { where } \sigma_{\mathrm{Rlh}}(\lambda) \cong \frac{128 \pi^{5} \zeta^{2}}{3 \lambda^{4}} \cdot \frac{6+3 \delta}{6-7 \delta} \text { for } \zeta=\frac{n-1}{2 \pi N_{0}}
$$

where $N_{0}$ is the number of particles per unit volume and $\delta$ is the polarization factor. $\delta$ is small and in the range from about 0.03 to 0.09 .

The two-layer aerogel RICH detector of the Belle II spectrometer [41] will separate charged particles in the forward end-cap of the spectrometer inside a magnetic field of $1.5 \mathrm{~T}$ with a high separation capability in the momentum range from 0.5 to $3.5 \mathrm{GeV} / c$. See Fig. 7.13. The detector will be ready for data taking in 2018.

\footnotetext{
${ }^{11}$ Opacity is another term for the mass attenuation coefficient or, depending on context, mass absorption coefficient. $\kappa_{\lambda}$ at a particular wavelength $\lambda$ of the electromagnetic radiation.
} 


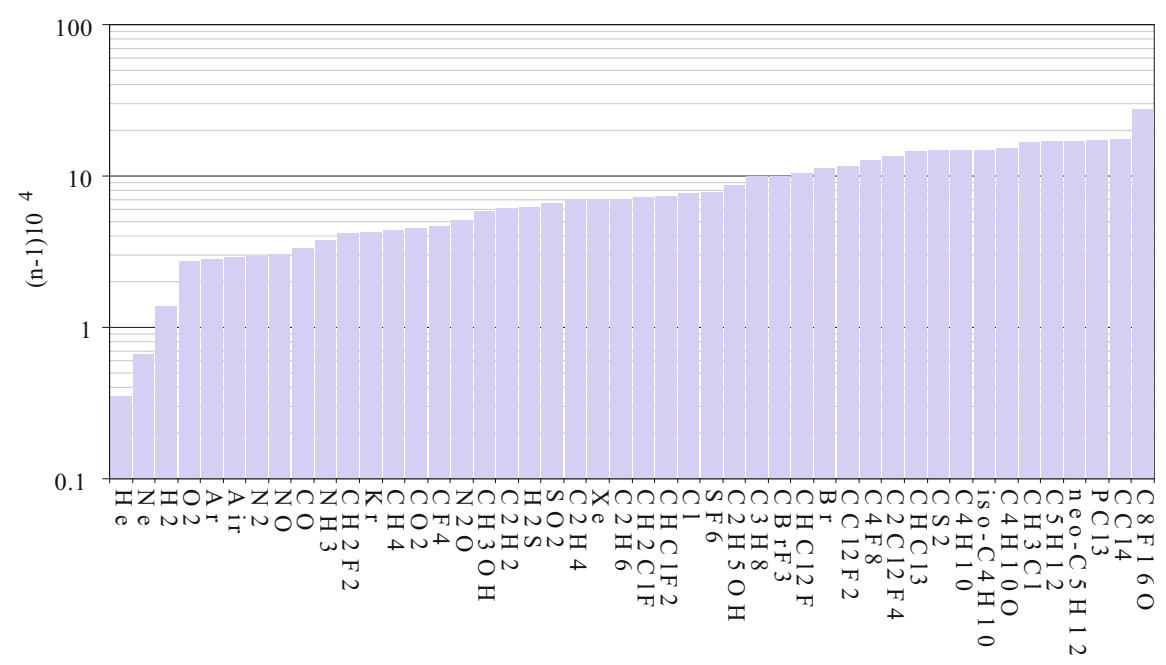

Fig. 7.14 Refractive index for some common fluids. D-line (589 nm). Data from [16, 22]

\subsubsection{Fluids as Radiators}

The relationship between the refractive index of a gas and the corresponding liquid, is given by:

$$
\left[\frac{n^{2}-1}{n^{2}+2}\right]_{\mathrm{gas}}=\left[\frac{p}{R T}\right]_{\mathrm{gas}}\left[\frac{M}{\rho}\right]_{\mathrm{liq}}\left[\frac{n^{2}-1}{n^{2}+2}\right]_{\mathrm{liq}}
$$

where $p$ and $T$ is the pressure and temperature of the gas, $M$ and $\rho$ is the molecular weight and density of the liquid and $R$ is the gas constant (based on pressure and volume units $\left.R=82.0575\left(\mathrm{~cm}^{3} \mathrm{~atm}\right) /(\mathrm{K} \mathrm{mol})\right)$.

The refractive index for a number of fluids is plotted in Fig. 7.14.

\subsubsection{Threshold Cherenkov Detectors}

As soon as photon detectors, Chap. 3, coupled with the associated electronics, had the sensitivity to detect the low level of photons emitted through Cherenkov radiation, the first threshold Cherenkov detectors, see Figs. 7.15 and 7.16, were used in high energy experiments. The best known of these early experiments, is probably the discovery of the antiproton at the Radiation Laboratory of the University of California at Berkeley in 1955 [42].

The design of these threshold detectors is simple as is shown in Fig. 7.16a. In this sketch, the radiator is a gas. There is no problem to change it by inserting 


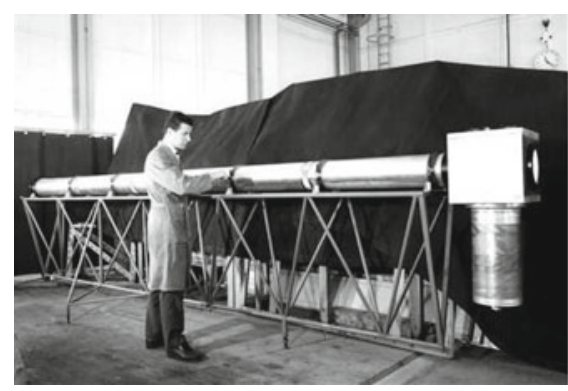

(a)

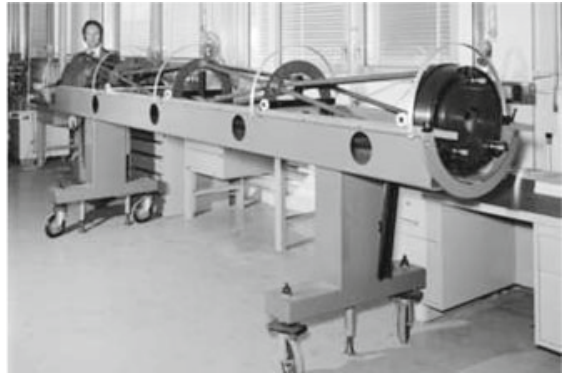

(b)

Fig. 7.15 (a) A threshold gas Cherenkov counter as used to tag particles in the secondary beams. CERN IT 6304088. (b) CEDAR counter (internal part). Here on the mounting bench. The counter is a differential Cherenkov, corrected for chromaticity, able to differentiate pions from kaons up to $350 \mathrm{GeV}$. Counters of this type were used in all SPS hadron beams. CERN PHOTO 7603033
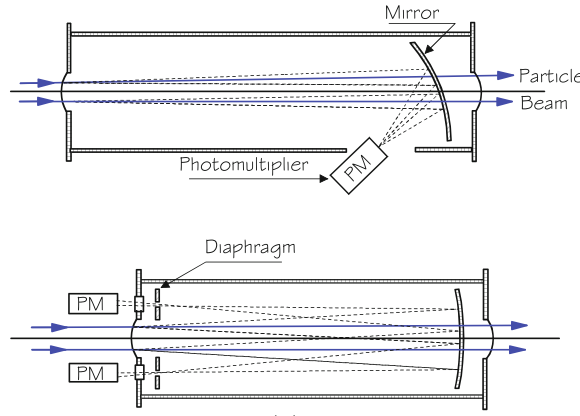

(a)

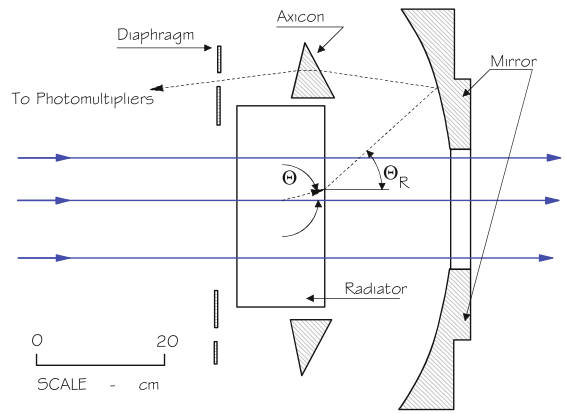

(b)

Fig. 7.16 (a) Top and bottom shows the working principles of respectively a threshold and a differential Cherenkov detector. (b) Is an achromatic liquid differential Cherenkov detectors, DISC: Differential Isochronous Self-Collimating; adapted from [43]

a solid or a liquid radiator, nor to change the pressure of the gas. It only affects the radiation length seen by the traversing particles. The solid angle covered by the detector is only limited by the design of the optics. A threshold Cherenkov detector can therefore be used both in the incoming beam to define the flavour of the primary particles as well as for identifying the secondaries. It should be noted that by introducing two, or more, detectors in series, positive particle identification can be achieved over a large momentum range.

A differential Cherenkov detector is shown in Fig. 7.16a. It is designed for a given value of the Cherenkov angle, such that:

$$
\Theta=r / F
$$

where $r$ is the mean radius of the aperture of the diaphragm and $F$ is the focal length of the mirror. The use of these detectors is mainly limited to parallel beams. 
Assuming high energy particles and gas radiator, the resolution power can be written as:

$$
\left[\frac{\Delta \beta}{\beta}\right]_{\text {limit }}=\tan \Theta \cdot \Delta \Theta
$$

The coma ${ }^{12}$ is the main error, given by:

$$
\Delta \Theta_{\text {coma }}=\Theta^{3}+\frac{\Theta^{2}}{4}\left[3 \frac{b}{L}-\Theta\right]=\frac{3}{4} \Theta^{3} \quad \text { if } \quad b \ll L
$$

where $b$ is the diameter of the incoming particle beam and $L$ is the length of the gas radiator. The chromatic angular dispersion is given by:

$$
\Delta \Theta_{\text {chrom }}=\frac{\Theta}{2 v}\left[1+\frac{1}{\gamma^{2} \Theta^{2}}\right] \quad \text { where } \quad v=\frac{n\left(\lambda_{2}\right)-1}{n\left(\lambda_{1}\right)-n\left(\lambda_{3}\right)},
$$

representing the optical dispersion in the gas. $\lambda_{1}$ and $\lambda_{3}$ are the wavelengths appropriate for the limits of the spectral range. $\lambda_{2}$ is the mean wavelength. The total angular dispersion is then:

$$
\Delta \Theta \approx \Theta^{3}+\frac{\Theta}{2 v}\left[1+\frac{1}{\gamma_{i}^{2} \Theta^{2}}\right]
$$

$i=0,1$ depending on the particle. We then get the limit for the maximum Cherenkov angle:

$$
\Theta^{4}+\frac{\Theta^{2}}{2 v} \leq \frac{1}{2 p^{2}}\left[m_{1}^{2}-m_{0}^{2}-\frac{m_{i}^{2}}{v}\right]
$$

For most applications, the Cherenkov angle will be smaller than this limit. The design will therefore be governed by the chromatic error.

To further diminish the errors, and thereby minimize $\Delta \beta / \beta$, a Differential Isochronous Self-Collimating, DISC, Cherenkov detector can be used. See Fig. 7.16b. With an optimized optics design a nearly achromatic condition can be achieved. That is,

$$
\frac{\Delta \Theta(\lambda)}{\Delta \lambda}=0 \rightarrow \frac{\Delta \beta}{\Delta \lambda}=0
$$

\footnotetext{
12 The aberration known as coma affects rays from points not on the axis of a lens. It is similar to spherical aberration in that both arise from the failure of the lens to image central rays and rays through outer zones of the lens at the same point. Coma differs from spherical aberration in that a point object is imaged not as a circle but as a comet-shaped figure (whence the term coma).
} 


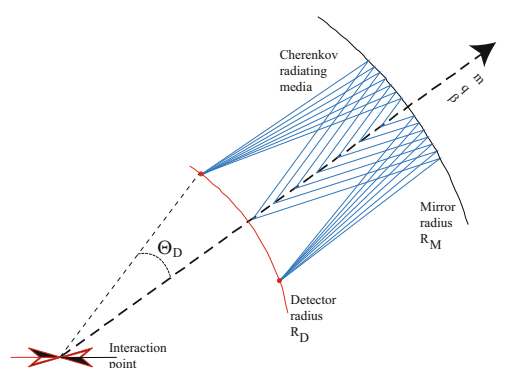

(a)

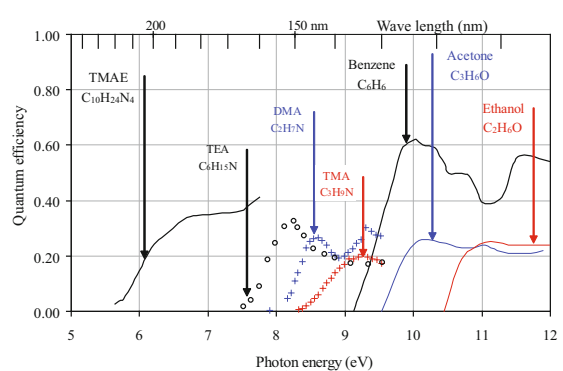

(b)

Fig. 7.17 (a) Ring imaging optics for particles emerging from a target or interaction region with zero impact parameter. The detected and emitted Cherenkov angles $\left(\Theta_{D}, \Theta\right)$ are equal if the detector radius is correctly chosen.[45]. (b) The quantum efficiency for some photo sensitive vapours as function of photon energy. Adapted from [17]

Velocity resolution $\Delta \beta / \beta \sim 10^{-6}-10^{-7}$ has been achieved [44]. These are very beautiful detectors, but with a somewhat limited usage as they require a near parallel beam, offer a limited solid angle and the material budget is not negligible.

\subsubsection{Ring Imaging Cherenkov Detectors}

The quest to make a ring imaging detector and thereby utilize all the inherent properties of Cherenkov radiation as described in Sect.7.4, was long thwarted by the inability to get a high spatial resolution photon detector which was sensitive to single photons and compatible with photon absorptions, Fig. 7.8, in the media and photon transmission through windows, Fig. 7.9b. The breakthrough came in 1977 with the work of J. Séguinot and T. Ypsilantis [45, 46]. See Fig. 7.17a. Their work during the initial phase was mainly concentrated around MWPC, Chap. 4, and a photoionizing vapour additive to the chamber gas.

\subsubsection{Photo Sensitive Vapours}

Figure $7.17 \mathrm{~b}$ shows the quantum efficiency for some photo sensitive vapours. The work with TEA, ${ }^{13}$ Triethylamin $\mathrm{C}_{6} \mathrm{H}_{15} \mathrm{~N}$, and especially TMAE, ${ }^{14}$ Tetrakis(dimethylamino)-ethylene $\mathrm{C}_{10} \mathrm{H}_{24} \mathrm{~N}_{4}[47,48]$, made it possible to work in the wavelength range from about 200 to $160 \mathrm{~nm}$ and thereby use fused silica as windows.

\footnotetext{
${ }^{13} \mathrm{http} / / /$ webbook.nist.gov/cgi/cbook.cgi?ID=C121448\&Units=SI.

${ }^{14} \mathrm{http}: / /$ webbook.nist.gov/cgi/cbook.cgi?ID=C996703\&Units=SI.
} 


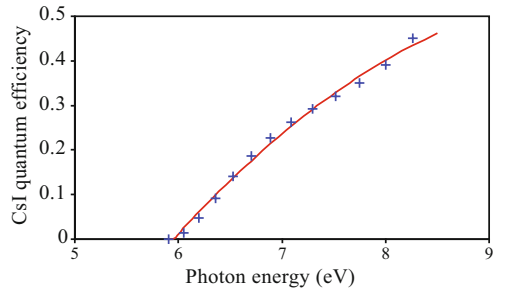

(a)

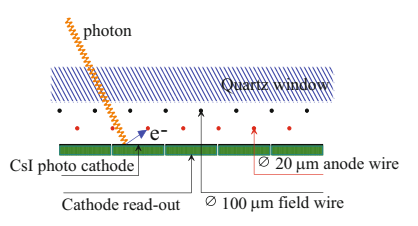

(b)

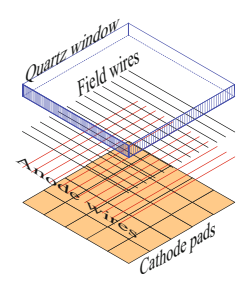

Fig. 7.18 (a) CsI quantum efficiency. (b) Sketch of a MWPC with CsI photo cathode

TMAE was the chosen photoionizing vapour, together with drift chambers, Chap. 3, for the first generation RICH detectors [49-51]. However, these fluids are difficult to handle and their usage is therefore now very limited. TEA and TMAE are chemically not reactive with respect to normal MWPC gases. They will, however, require an $\mathrm{O}_{2}$ and water content of the carrier gas $\leq 10 \mathrm{ppm}$ for stable operation. A drawback by using these molecules is the photon feedback. The photons created in the gas amplification process have a probability to convert. The main source of this background is from the ionization due to the charged particle going through the detector. The chambers were normally run at an amplification around $1-5 \cdot 10^{5}$ in order to be sensitive to single photons. The total probability for re-conversion thereby became larger than 1 and the chamber would break down. The number of feed-back photons can be written as $N_{\mathrm{fp}}=\iota \cdot G$ where $G$ is the total chamber gain. ${ }^{15}$ $\iota \sim 7-8 \cdot 10^{-6}$ in $\mathrm{CH}_{4}$ due to photon absorption for wavelengths below $143 \mathrm{~nm}$. See Eq. (7.21).

A number of ingenious chamber designs were made to minimize the photon feed-back. The designs are a compromise between detection efficiency, ease of operation and fabrication and drift of electrons in a $\mathbf{B} \times \mathbf{E}$ configuration. Even at stable operating conditions, some photons will escape and give rise to an event correlated background. This background is difficult to disentangle from the real signal in high occupancy events and particularly with TMAE due to its long photon conversion length.

\subsubsection{CsI Photo Cathode}

The next step in high spatial granularity, or pixilated, photon detectors for RICH came with the CsI photon detector [52]. CsI is an alkali halide crystal which has a good quantum efficiency, Fig. 7.18a, below $200 \mathrm{~nm}$ and is stable in normal dry and $\mathrm{O}_{2}$ free chamber gases [53]. The development was triggered by the need for a faster detector at the arrival of LHC and similar accelerators. In a MWPC structure,

\footnotetext{
15 The measured chamber gain might be smaller due to charge sharing and electronics time constants.
} 
the CsI photo cathode can either be deposited as a reflective, Fig. 7.18b, or as a semi-transparent layer [54]. The latter would, in the case of Fig. 7.18b lay-out, be a layer on the quartz window. The maximum quantum efficiency for semitransparent CsI is for a thickness of about $11 \mathrm{~nm}$ in the wavelength range from 210 to $170 \mathrm{~nm}$. The thickness does not matter for a reflective photo cathode and is normally in the range of $150-200 \mathrm{~nm}$. A semi-transparent CsI photo cathode will have a quantum efficiency of about 0.7 compared to a reflective one. It should be noted that the photon conversion efficiency is strongly depending on the bulk structure and morphology of the CsI layer; that is, the roughness of the substrate and the connectivity of the layer. Particularly thin layers can become a collection of unconnected islands. Post-production heat treatment has proven advantageous.

As for the photosensitive vapours, a CsI photo cathode will be sensitive to the photon feed-back from the gas amplification process, see Sect.7.4.4.1. A stable operation of the chamber is therefore a compromise between single photon efficiency, electronics sensitivity, signal shaping and gas amplification.

As few, if any other photon detector, can beat a gas based detector in cost efficiency and geometrical acceptance, a number of similar, but not identical, detector set-ups are proposed and investigated. The main emphasis is on limitation of photon feed-back, on better and more stable photo cathodes and on time resolution. This work is also partially driven by very large Cherenkov detectors for astrophysics. A very promising research and development is in gaseous micro pattern detectors with Bialkali photo cathodes. We will not discuss these here, but refer the reader to [55]. An overview of the current status and perspectives of gaseous photon detectors can be found in [56].

\subsubsection{Vacuum Based Photon Detectors}

The working principles of vacuum based photon detectors like photo multiplier tubes are discussed in Chap. 3. Although small diameter PM tubes, diameter $10 \mathrm{~mm}$ upwards, have been used for a long time in Cherenkov detectors, cost, balanced with space resolution and material budget, made them less attractive. The introduction of multi anode and pixilated silicon anode detectors, together with fast and sensitive electronics changed this. The first generation of multi anode photo tubes required a lens system [57] in order to give good geometrical acceptance. See Fig. 7.19a.

The schematic of a Hybrid Photon Detector [60, 61], HPD, is shown in Fig. 7.19b. In these detectors the encapsulated pixilated silicon detector is bump-bonded onto the read-out electronics. The capacitance is thereby small and the associated noise low. It also requires only a few vacuum feed-throughs. The photo cathode is normally a S20. ${ }^{16}$ Under the influence of the electric field, the photo-electron is accelerated onto the silicon detector. In the example given in Fig. 7.19b, the $20 \mathrm{kV}$ potential between anode and cathode gives a cross-focusing field with a

${ }^{16} \mathrm{~S} 20$ is a tri-alkaline (Sb-Na-K-Cs) semi-transparent photo cathode. 


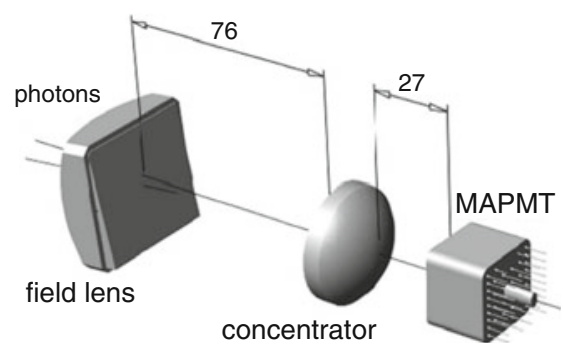

(a)

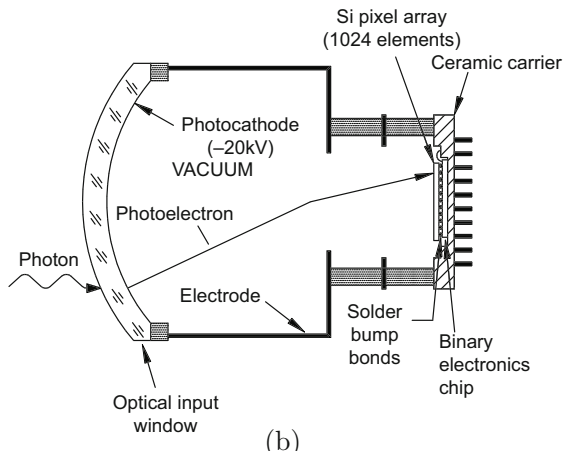

(b)

Fig. 7.19 (a) Optical arrangement of the COMPASS MAPMT and the fused silica lens telescope. With permission [58]. (b) Schematic arrangement of the LHCb Hybrid Photon Detector. With permission [59]

demagnification of $\sim 5$. Other field configurations can be used [61]. The granularity of the silicon detector can be tailored as function of the required geometrical resolution.

These new photon detectors with a maximum quantum efficiency of about 30 $35 \%$ around $300 \mathrm{~nm}$, have made the choice of Cherenkov radiators and photon windows much more flexible. It has for instance allowed the use of aerogel in Ring Imaging Cherenkov detectors. See Sect. 7.4.2.2 and Fig. 7.12c.

Current research and development is mainly concentrated on faster and cheaper detectors with large geometrical acceptance. These are detectors like silicon avalanche photo diodes, micro channel plates and large area flat panel multi-anode PMTs. The reader is referred to Chap. 3.

An overview of the current status and perspectives of vacuum-based photon detectors can be found in [62].

\subsubsection{Optics}

We can broadly divide the light collection system of Ring Imaging Cherenkov detectors into two distinctive classes.

- Proximity focusing, or direct light collection as in Fig. 7.20.

- Concave mirrors as in Fig. 7.17a in Sect. 7.4.4.

\subsubsection{Proximity Focusing}

In the first case with proximity focusing optics, the resolution relies on the thinness, 1 , of the radiator in comparison to the expansion length, $\mathrm{L}$. That is, $1 \ll \mathrm{L}$. The Cherenkov light will then describe a thin cone around the charged particle and 
Fig. 7.20 Proximity focusing arrangement

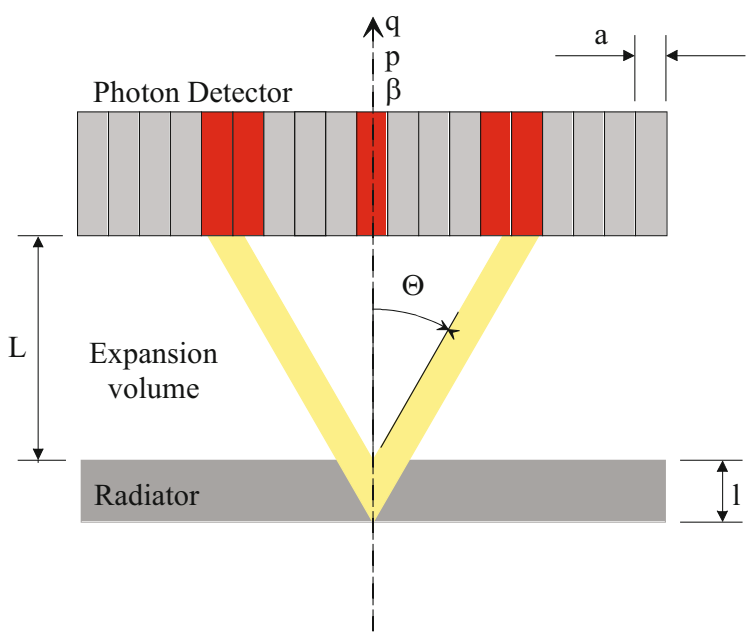

will give rise to a finite width, conic section image where the detector plane intercepts this cone. If the particle is not perpendicular to the radiator and the detector planes, this circular image becomes distorted to an elliptic or a hyperbolic image. Depending on the refractive index of the radiator, photon window material and the expansion gap, light might be trapped due to total internal reflections. See Sect.7.4.2.1. As the photon detector has to be placed in the path of the charged particle, the material budget may become prohibitive. However, this detector configuration is well adapted to $4 \pi$ detectors with high refractive index radiators $[51,63]$.

\subsubsection{Focusing Mirrors}

Detectors which cover large solid angles require large focusing mirrors as in Fig. 7.21. There are two. ${ }^{17}$ options, parabolic [65] and spherical [66, 67] mirror. The choice of mirror substrate is a balance between cost, ease of fabrication and performance. Whereas the material budget is normally not an issue in astrophysics, see Sect. 6.1 and Fig. 7.21a and b, it is one of the main concerns in accelerator based experiments as the mirrors must be inside the acceptance. If spherical aberration becomes a dominant contribution to the total error in the Cherenkov angle calculation, parabolic mirrors should be used.

\footnotetext{
${ }^{17}$ We will not discuss here ellipsoid nor hyperbolic mirrors. For correctors like Schmidt and Maksutov, the reader is referred to [40].
} 


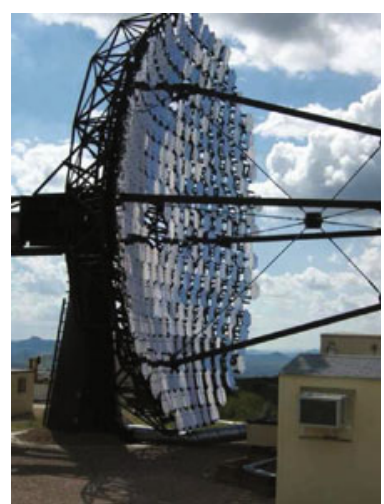

(a)

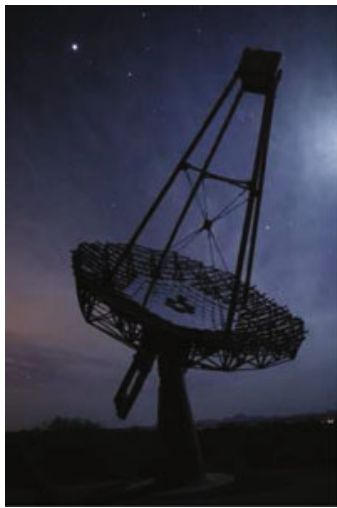

(b)

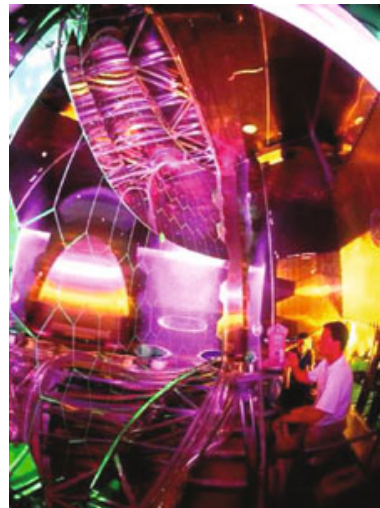

(c)

Fig. 7.21 (a) and (b) The VERITAS Telescope 1 as installed at the Whipple Observatory base camp. The collector dish has a diameter of $12 \mathrm{~m}$ and a focal length of $12 \mathrm{~m}$ and comprises 350 mirror facets. A 499-PMT camera is installed in the box at the focal point. Courtesy of the VERITAS Collaboration [64]. (c) COMPASS [67] mirror wall of RICH 1. CERN EX 0106007 01

Table 7.3 Basic material properties for some mirror substrates together with substrate rigidity, $K$, and the rigidity divided by material thickness in units of radiation length

\begin{tabular}{l|l|l|l|l|l}
\hline Material & $\begin{array}{l}\mathrm{X}_{0} \\
{[\mathrm{~cm}]}\end{array}$ & $\begin{array}{l}E \\
{\left[10^{4} \mathrm{MPa}\right]}\end{array}$ & $\begin{array}{l}\alpha \\
{\left[10^{-6} /{ }^{\circ} \mathrm{C}\right]}\end{array}$ & $\begin{array}{l}\text { Relative } \\
\text { rigidity } K\end{array}$ & $\begin{array}{l}K / X_{0} \\
\text { relative }\end{array}$ \\
\hline Beryllium & 35.3 & 28.9 & 11.3 & 1 & 1 \\
\hline Plexiglas & 34.4 & 0.33 & 70 & 0.012 & 0.011 \\
\hline Pyrex glass & 12.7 & 6.17 & 3.2 & 0.213 & 0.076 \\
\hline Aluminium & 8.9 & 6.9 & 23.9 & 0.238 & 0.060 \\
\hline
\end{tabular}

$X_{0}$ is the radiation length, $E$ the Young's module and $\alpha$ is the coefficient of thermal expansion

The material option for the mirror substrate is a balance between radiation length, size of the substrate and stability. Some options ${ }^{18,19}$ are given in Table 7.3.

The rigidity, $K$, of a thin mirror substrate is roughly given by:

$$
K \propto \frac{E t^{3}}{D^{2}}
$$

where $E$ is Young's modulus, $t$ is the substrate thickness and $D$ is the diameter. The superiority of substrate materials like beryllium is clear in Table 7.3. In this table

\footnotetext{
${ }^{18}$ Plexiglas is Poly(methyl methacrylate) (PMMA) by Evoniks Business Unit Performance Polymers.

${ }^{19}$ Pyrex, Corning Incorporated, is made of $4 \%$ boron, $54 \%$ oxygen, $3 \%$ sodium, $1 \%$ aluminium, $38 \%$ silicon, and less than $1 \%$ potassium.
} 
substrates of diameter 500 and $5 \mathrm{~mm}$ thickness are compared. However, beryllium is not a good reflector nor a good support for a reflecting surface. A thin glass face is therefore required on the beryllium as support for the reflector [68]. This glass surface can also be used to adjust the focal length of the mirror. The main challenge is to use a glass which has the same thermal expansion coefficient as beryllium.

Thin and robust mirror substrates can be made as a sandwich assembly. The kernel is normally a honeycomb or foam and the inner and outer skin are preformed to about the right radius of curvature. The final adjustment is done at the assembly stage or by reshaping, by polishing, the reflecting skin later. The skin can be high strength carbon fibre sheets [69], easily formed Plexiglas [70] or simple metal structures [71]. Glass with glass-foam kernel has also been built [72].

Glass is still the most used substrate for mirrors. It is easily shaped and machined and the ageing behaviour is well known. Stresses in the material can be simply relieved. It is also inert in most Cherenkov radiators. It is normally slumped to the required shape and then polished to the final focal length. Its principal drawback is the radiation length.

\subsubsection{The Reflective Surface}

The reflectivity of a surface is a function of the incident angle and energy of the light and the dielectric structure of the surface. The principle is discussed in [40] and more specifically in [73]. See Fig. 7.22a. A high reflectivity layer is over-coated by one or more transparent films of high and low refractive indices. Aluminium and silver are good reflectors with peak reflectivity of respectively $\sim 92 \%$ and $\sim 96 \%$. Aluminium, the most widely used metal for reflecting films, offers consistently high reflectance throughout the visible, near-infrared, and near-ultraviolet regions of the spectrum.

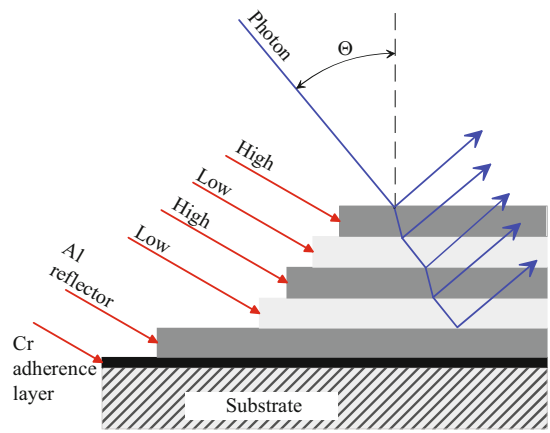

(a)

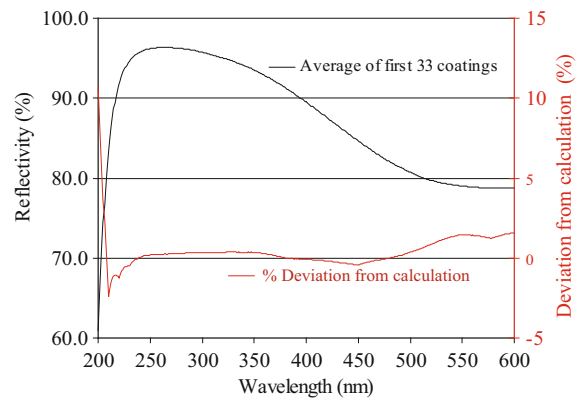

(b)

Fig. 7.22 (a) Schematic representation of a metal multi-dielectric mirror [73]. (b) Measured and calculated reflectivity of a multi-dielectric mirror coating. The stack is $\mathrm{Cr}-\mathrm{Al}-\mathrm{SiO}_{2}-\mathrm{HfO}_{2}$. Adapted from [73] 
Table 7.4 Typical process parameters for a multi-dielectric mirror coating [73]

\begin{tabular}{l|l|l|l|l}
\hline Material & $\begin{array}{l}\text { Purity } \\
{[\%]}\end{array}$ & $\begin{array}{l}\text { Chamber pressure } \\
{[\mathrm{Pa}]}\end{array}$ & $\begin{array}{l}\text { Deposition rate } \\
{[\mathrm{nm} / \mathrm{s}]}\end{array}$ & $\begin{array}{l}\text { Thickness (geom.) } \\
{[\mathrm{nm}]}\end{array}$ \\
\hline $\mathrm{Cr}$ & 99.98 & $2 \cdot 10^{-5}$ & 1 & 20 \\
\hline $\mathrm{Al}$ & 99.999 & $2 \cdot 10^{-5}$ & 5 & 85 \\
\hline $\mathrm{SiO}_{2}$ & 99.99 & $10^{-3} \mathrm{O}_{2}$ & 0.2 & 28 \\
\hline $\mathrm{HfO}_{2}$ & 99.9 & $10^{-3} \mathrm{O}_{2}$ & 0.2 & 38 \\
\hline
\end{tabular}

While silver exhibits slightly higher reflectance than aluminium through most of the visible spectrum, the advantage is temporary because of oxidation tarnishing. Aluminium also oxidizes, though more slowly, and its oxide is tough and corrosion resistant. Oxidation significantly reduces aluminium reflectance in the ultraviolet and causes slight scattering throughout the spectrum. Generally, all reflective layers need a protective film.

Material like $\mathrm{SiO}_{2}$ and $\mathrm{MgF}_{2}$ have low refractive index in comparison to $\mathrm{HfO}_{2}$ and $\mathrm{TiO}_{2}$. Properties like residual stress, adherence, resistivity to abrasion and humidity and coating yield are essential in the selection process for these layers. The optical thickness of the layers, $d_{\text {opt }} \propto \cos \Theta$, is normally chosen to be $\lambda / 4$. A dielectric coating will lead to a wavelength and angle dependent modulation of the reflectivity. The larger the ratio between the refractive indices in a Low/High pair, the higher is the peak reflectivity and width of the enhanced region. Adding more pairs for the same wavelength range, will enhance the peak reflectivity, but narrow the wavelength range. The layer stack will normally be terminated with a high refractive index layer. In this way the mirror reflectivity can be optimized for the wavelength range of the photon detector.

Mathematically approximation codes ${ }^{20}$ will predict the behaviour of the multilayer film. The accuracy only depends on the knowledge of the refractive index and the absorption in the deposited layers. These optical properties are however dependent of the deposition method and processing parameters.

An example is shown in Fig. 7.22b. Layers of $\mathrm{Cr}, \mathrm{Al}, \mathrm{SiO}_{2}$ and $\mathrm{HfO}_{2}$ are used on a glass substrate. See Table 7.4 for process parameters. This coating is optimized for a wavelength of $275 \mathrm{~nm}$ in order to match a S20, footnote 16, photo cathode and compared with calculations. See footnote 20 for the calculation.

\subsubsection{Mirror Imaging Quality}

The error introduced by the imaging quality of a RICH mirror should be small compared to all other errors in the detector. If the mirror is a perfect spherical

\footnotetext{
${ }^{20}$ FilmStar Design, FTG Software Associates, Princeton, NJ, SCI Film Wizard, Scientific Computing International, Carlsbad, CA or similar.
} 
Fig. 7.23 (a) Spot image for a high precision glass mirror. (b) Spot image for a thin glass mirror [66]

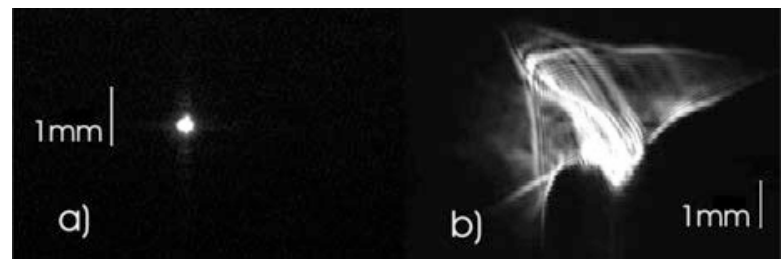

surface, the spot on the focal plane would have the size given by the diffraction limit. For a circular mirror of diameter $D$ and a radius of curvature $R$, the diffraction limited spot diameter, $d$, at the third maximum, corresponding to $95.3 \%$ of the focused light, is given by:

$$
d=2 R \tan \alpha \quad \text { for } \quad \sin \alpha=\frac{\lambda x}{\pi D} \quad \text { and } \quad x=3.7 \pi
$$

For a wavelength $\lambda=641 \mathrm{~nm},{ }^{21} D=0.50 \mathrm{~m}$ and $R=8 \mathrm{~m}, d=76 \mu \mathrm{m}$.

Real mirrors have real imperfections. Fig. 7.23 shows the difference between a high precision and a thin glass mirror. The mirror in Fig. 7.23a is a $50 \mathrm{~mm}$ thick glass mirror of diameter $400 \mathrm{~mm}$ and a radius of curvature of $7.8 \mathrm{~m}$. The Fig. $7.23 \mathrm{~b}$ mirror is $7.5 \mathrm{~mm}$ thick with a diameter of $400 \mathrm{~mm}$ and a radius of curvature of $7.8 \mathrm{~m}$. $95 \%$ of the focused light for the first mirror is inside circle of diameter $0.23 \mathrm{~mm}$. The corresponding diameter for the second mirror is $3.4 \mathrm{~mm}$. This mirror also features irregularities at the edges of the surface. The average quality of a mirror is well described by the spot size at the focal plane and is normally sufficient as a qualification parameter. Let $D_{0}$ be the diameter of this spot which encompasses $95 \%$ of the light. $\sigma_{\mathrm{s}}=D_{0} / 4$ is the RMS of the light distribution if this distribution was Gaussian. The error induced by the mirror is then given by:

$$
\sigma_{\Theta}=\frac{\sqrt{\sigma_{\mathrm{s}}^{2}+\sigma_{\mathrm{p}}^{2}}}{2 R} \approx \frac{\sigma_{\mathrm{s}}}{2 R}=\frac{D_{0}}{8 R}
$$

where $\sigma_{\mathrm{p}}$ is the resolution of the point source.

The determination of the spot shape can be an invaluable tool in the development and fabrication process. The quantification of the variation in the radius of curvature across a substrate can be used to improve the resolution of the system. It can be particularly important for large mirrors.

Shack-Hartmann sensors, Ronchi test method, Foucault method and similar measurement methods are described in detail in [74]. We will only show the power of these methods with one example.

A sketch of a Ronchi test set-up is shown in Fig. 7.24a. A beam of coherent, quasi-monochromatic light is brought to focus by an optical system that is under-

\footnotetext{
${ }^{21}$ Red laser diode.
} 


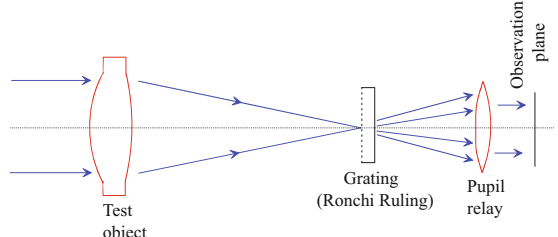

(a)

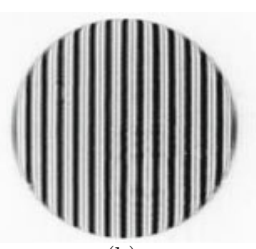

(b)

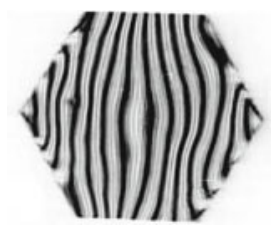

(c)

Fig. 7.24 (a) General set-up for a Ronchi test. (b) Ronchigram of a high precision spherical glass mirror. Thickness $50 \mathrm{~mm}$. (c) Ronchigram of a thin spherical glass mirror. Thickness $4.5 \mathrm{~mm}$. Ronchi ruling $1 \mathrm{~mm}$

going tests to determine its aberrations. A lens, or more generally any optical system consisting of an arrangement of lenses and mirrors, is placed in the position Test Object. A diffraction grating, placed perpendicular to the optical axis in the vicinity of the focus, breaks up the incident beam into several diffraction orders. The diffracted orders propagate, independently of each other, and are collected by a pupil relay lens, which forms an image of the exit pupil of the object under test at the observation plane. For a concave mirror, deviation from a spherical surface will result in deformation of the fringes. The measurement is only sensitive to changes in radius of curvature perpendicular to the grating direction. Results are shown in Fig. 7.24. Figure 7.24b is a Ronchigram for a high precision spherical mirror, whereas Fig. 7.24c is for a thin large mirror. For the first mirror, the interference lines are straight which shows that the deviation from the ideal shape is smaller than the resolution of the Ronchi ruling. For the second mirror, the interference lines are distorted. In the centre, the lines bow outward and indicate parabolic deformation. On the edges, the lines bow inward to indicate an oblate spheroid surface.

\subsubsection{Ring Finding and Particle Identification}

As explained in Sect. 7.4, Cherenkov light is produced in a cone at polar angle $\Theta_{\mathrm{C}}$ relative to the particle trajectory, as given by Eq. (7.8) for a particle travelling at velocity $\beta$. In a RICH detector the light is focussed onto a detector plane as a ring image. For the classical RICH geometry illustrated in Fig. 7.17a and [46], the detected photons corresponding to a track passing through the detector would form a circular ring image centred on the track impact point on the detector. The issues discussed in this section are the finding of the ring, i.e. the pattern recognition to associate the detected photons to a given track, and the particle identification, i.e. the determination of the particle type, given the photons that are associated to its track. Examples are taken from LHCb, Fig. 7.25, the dedicated B physics experiment at the LHC, which has two RICH detectors [75]. A review of other approaches can be found in [76]. 


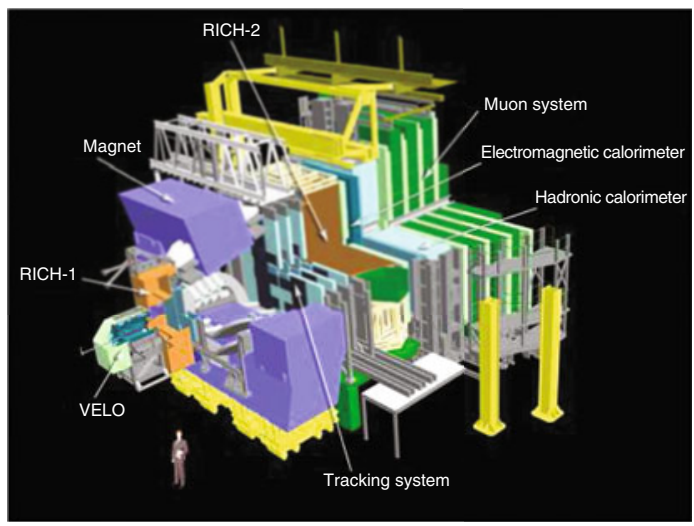

(a)

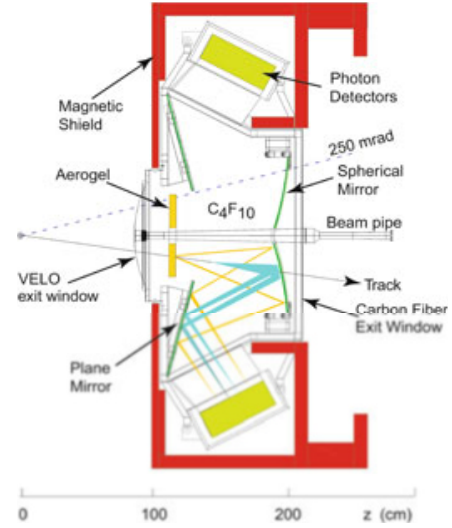

(b)

Fig. 7.25 (a) View of the LHCb detector. (b) Side view schematic layout of the RICH 1 detector. Reference [75]

For the simple detector geometry of Fig.7.17a, and for a single track passing through the detector, the circular image implies that the photons from the track all lie at a constant radius on the detector plane, when measured from the track impact point. The radius $r$ is related to the Cherenkov angle, $\Theta_{\mathrm{C}}$, by:

$$
r=R \Theta_{\mathrm{C}} / 2
$$

where $R$ is the radius of curvature of the spherical focussing mirror. For a given track the pattern recognition could therefore simply be performed by plotting the radius of all photons in this way, and searching for a peak in the distribution. Due to the finite resolution, this signal peak will have a roughly Gaussian shape, with width corresponding to the resolution. Sources of finite resolution include the pixel size of the photon detector, and the fact that the refractive index has some dependence on the photon wavelength, leading to a chromatic term in the resolution. Background hits that are distributed randomly across the detector plane, for example from noise in the photon detector, will appear as a contribution in the plot of detected photon radius that increases roughly linearly with radius (due to the increasing area swept out on the detector plane as the radius increases). This situation is illustrated in Fig. 7.26a.

Given the reconstructed radius $r$, the Cherenkov angle can be calculated from Eq. (7.38), and thus the velocity $\beta$ of the particle determined from Eq. (7.8). To make the final step of identifying the particle, the momentum $p$ must also be known, usually from the tracking system of the experiment that measures the curvature of the track in a magnetic field. Then the mass $m$ of the particle can be determined using relativistic kinematics:

$$
m^{2}=p^{2}\left(\beta^{-2}-1\right) / c^{2}
$$




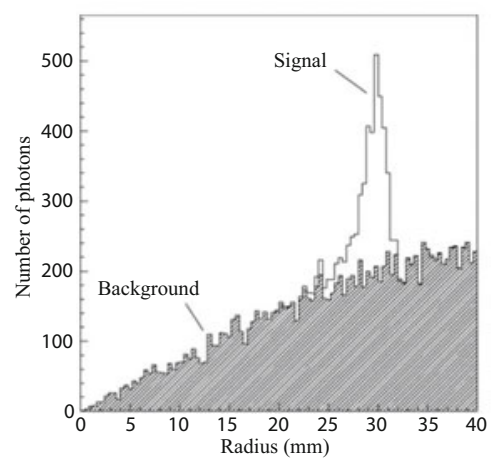

(a)

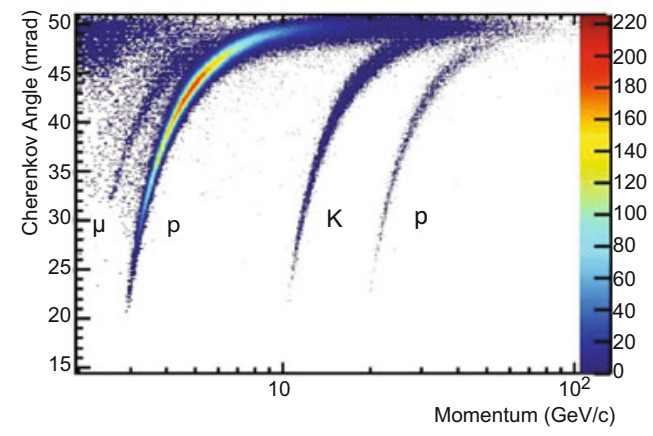

(b)

Fig. 7.26 (a) Distribution of photons in radius around the track, for a set of tracks in one of the LHCb RICH detectors; the peak from the photons associated to the track is visible, along with background from other sources. (b) Reconstructed Cherenkov angle for isolated tracks, as a function of track momentum in the $\mathrm{C}_{4} \mathrm{~F}_{10}$ radiator. The Cherenkov bands for muons, pions, kaons and protons are clearly visible. Reference [77]

and this identifies the particle type. An example is shown in Fig. 7.26b where the reconstructed Cherenkov angle has been plotted versus momentum for all the particles in a set of events, and the loci of points corresponding to particles with different masses are clearly seen.

In practical implementations of the RICH technique, the optical system usually differs from the simple classical layout, so as to avoid the material of the photon detectors being placed within the acceptance of the spectrometer. For example, the $\mathrm{RICH}$ detectors of the $\mathrm{LHCb}$ experiment involve a spherical focussing mirror that is tilted with respect to the track direction, and an additional planar mirror to bring the Cherenkov light to photon detectors sited outside the acceptance, while limiting the overall size of the detector system. This complicates the reconstruction somewhat, as the ring images are no longer circular but become distorted into roughly elliptical shapes, and the track no longer passes through the detector plane, but its image on that plane has to be calculated from knowledge of the optics. There is also an additional contribution to the resolution, due to the spherical aberration resulting from imaging the photons from off-axis tracks, but this can usually be arranged to be smaller than the limiting chromatic effect. The distortion of the ring image can be exactly corrected for by reconstructing the Cherenkov angle for each photon-track pair. For a spherical focussing mirror the analytical solution of this calculation involves the solution of a quartic equation. See [78]. For reasons of speed, a numerical approach can be used instead, ray-tracing photon candidates through the optical system and calibrating the distortion of the ring image in this fashion. The peak search is then performed in Cherenkov angle space, rather than radius on the detector plane. 


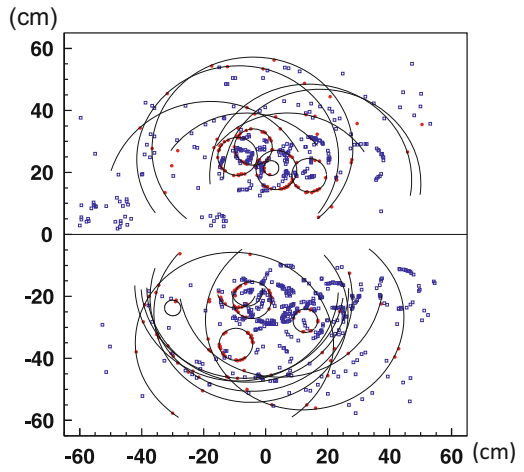

(a)

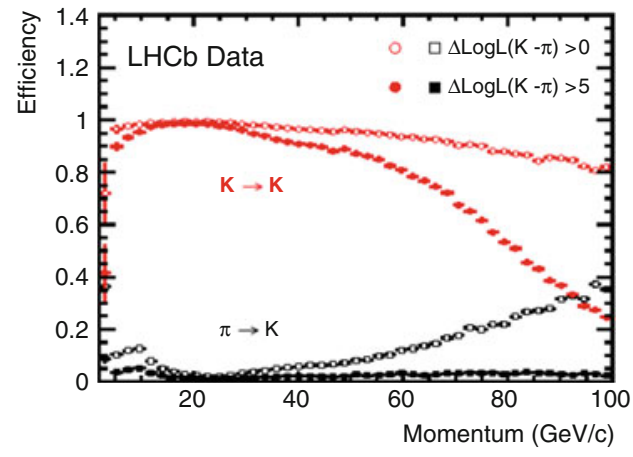

(b)

Fig. 7.27 (a) Ring images from tracks passing through the RICH 1 detector of $\mathrm{LHCb}$, from a single proton-proton collision event at the LHC. (b) Kaon identification efficiency and pion misidentification rate as measured using data. Two different $\Delta \log \mathcal{L}(\mathrm{K}-\pi)$ requirements have been imposed on the samples, resulting in the open and filled marker distributions, respectively. Reference [77]

This approach of peak searching works well in situations of low track multiplicity, where the ring images from tracks are well separated. However, at the LHC the track density is high, as illustrated for a typical event in Fig. 7.27a. In this case the main background to the reconstruction of the ring image of a given track comes from the overlapping rings from other tracks. It is therefore advantageous to consider the optimization of photon assignment to all of the tracks in the event simultaneously, in a so-called global approach. Since a momentum measurement is required to convert a measured ring image into particle identification, as discussed above, it makes sense to use the reconstructed tracks in the event as the starting point for pattern recognition. Trackless ring searches have been developed, but are mostly relevant for background suppression, rather than particle identification [76]. Furthermore, the number of stable charged particle types that are required to be identified is rather limited, typically five: e, $\mu, \pi, \mathrm{K}$, p. The pattern recognition can be made faster by just searching for these particle types, i.e. hypothesis testing. For applications where speed is crucial, such as use in the trigger of the experiment, the number of hypotheses compared can sometimes be further reduced, depending on the physics process that is being selected, e.g. simply comparing $\pi$ and K hypotheses [79]. On the other hand, if one is interested in an unbiased search for charged particles (such as exotic states) then alternative approaches exist that do not rely on preselected hypotheses [80].

The pattern recognition then proceeds by taking the most likely hypothesis for each of the tracks in the event, typically the $\pi$ hypothesis as they are the most abundantly produced particle (at the LHC). The likelihood is then calculated that the observed pattern of photons was produced by the particles, under these first choices of mass hypotheses. Conceptually this corresponds to taking the product of terms for each photon according to how close it is to the nearest ring image, assuming 


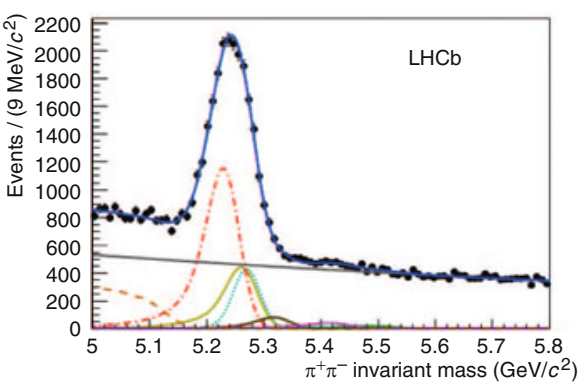

(a)

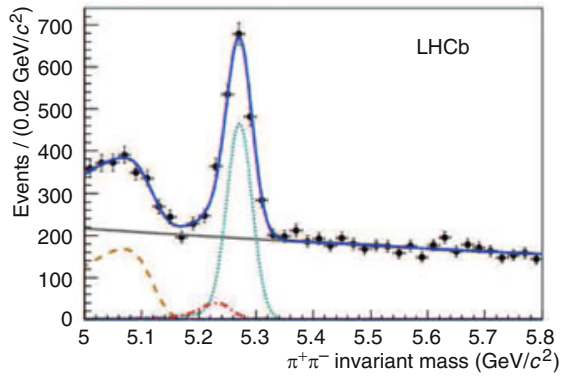

(b)

Fig. 7.28 (a) Invariant mass distribution for $\mathrm{B} \rightarrow h^{+} h^{-}$decays in the LHCb data before the use of the RICH information, and (b) after applying RICH particle identification. The signal under study is the decay $\mathrm{B}^{0} \rightarrow \pi^{+} \pi^{-}$, represented by the turquoise dotted line. The contributions from different b-hadron decay modes $\left(\mathrm{B}^{0} \rightarrow \mathrm{K} \pi\right.$ red dashed-dotted line, $\mathrm{B}^{0} \rightarrow 3$-body orange dasheddashed line, $\mathrm{B}_{s} \rightarrow \mathrm{KK}$ yellow line, $\mathrm{B}_{s} \rightarrow \mathrm{K} \pi$ brown line, $\Lambda_{b} \rightarrow \mathrm{pK}$ purple line, $\Lambda_{b} \rightarrow \mathrm{p} \pi$ green line), are eliminated by positive identification of pions, kaons and protons and only the signal and two background contributions remain visible in the plot on the right. The gray solid line is the combinatorial background. Reference [81]

a Gaussian probability distribution around each ring. A term is also added to the likelihood from the comparison of the total number of photons assigned to a track, compared to the expected number given the mass hypothesis and momentum. The tracks in the event are then all checked to see which would give the greatest increase in the total likelihood of the event, if its hypothesis were to be changed, and the mass hypothesis of the one giving the greatest increase is then changed. This procedure is iterated until no further improvement in the likelihood can be achieved, at which point the maximum-likelihood solution to the pattern recognition has been found. By the use of various computational tricks [78] this algorithm can be reasonably fast, typically taking a similar CPU time to the track finding algorithm. The performance of this approach to particle identification when applied to $\mathrm{LHCb}$ events (of the type shown in Fig. 7.27a) is illustrated in Fig. 7.27b. The efficiency for identifying kaons and the misidentification rate of pions are both shown as a function of momentum.

An example from the $\mathrm{LHCb}$ experiment of the resulting powerful particle identification in $\mathrm{B} \rightarrow h^{+} h^{-}$decays is shown in Fig. 7.28. The LHCb experiment moves to a fully software trigger where the RICH information is embedded.

\subsection{Transition Radiation Detectors}

A charged particle in uniform motion in free space will not radiate. It can radiate if it traverses a medium where the phase velocity of light is smaller than the velocity of the charged particle. This is Cherenkov radiation as discussed in Sect. 7.4 and was first correctly described by P.A. Cherenkov and S.I. Vavilov in 1934 and formulated 
by I.M Frank and I.E. Tamm in 1937 [15]. This radiation was worked into the BetheBloch formalism in 1940 by E. Fermi, see Chap. 2 and [82].

There is another type of radiation when the charged particle traverses a medium where the dielectric constant, $\varepsilon$, varies. This is transition radiation. It is analogous to bremsstrahlung. In both cases the radiation is related to the phase velocity of the electromagnetic waves in the medium and the velocity of the particle. In the case of transition radiation, the phase velocity changes whereas the particle velocity changes for bremsstrahlung. Transition radiation is, like bremsstrahlung, strongly forward peaked.

V.L. Ginzburg and I.M. Frank predicted in 1944 [83] the existence of transition radiation. Although recognised as a milestone in the understanding of quantum mechanics, transition radiation was more of theoretical interest before it became an integral part of particle detection and particle identification [84].

The exact calculation of transition radiation is complex and we will not repeat the mathematics here. The reader is referred to $[18,85,86]$. Specific discussions can be found in $[87,88]$. We will here just recall some of the central features.

Transition radiation is emitted when a charged particle traverses a medium with discontinuous dielectric constant. Let $\left[\mathbf{E}_{1}, \mathbf{H}_{1}\right]$ be the Lorentz transformed Coulomb field of the charged particle in medium 1 and $\left[\mathbf{E}_{2}, \mathbf{H}_{2}\right]$ the corresponding one in medium 2. See Fig. 7.29a. $\left[\mathbf{E}_{1}, \mathbf{H}_{1}\right]$ and $\left[\mathbf{E}_{2}, \mathbf{H}_{2}\right]$ do not match at the boundary. In order to satisfy the continuity equation, a solution of the homogeneous Maxwell equation must be added in each medium. This is the transition radiation. The angular distribution of transition radiation by a perfectly reflecting metallic surface is of the form:

$$
J(\Theta)=\omega \frac{d N}{d \omega d \Omega}=\frac{\alpha}{\pi^{2}}\left(\frac{\Theta}{\gamma^{-2}+\Theta^{2}}\right)^{2}
$$

where $\gamma=E / m \gg 1$ in natural units, $\hbar=c=1, \alpha \simeq 1 / 137$ is the fine structure constant and $\Theta \ll 1$ is the angle of the photon with respect to the velocity vector $\mathbf{v}$ of the charged particle. $\Theta$ is along $\mathbf{v}$ for forward transition radiation or its mirror

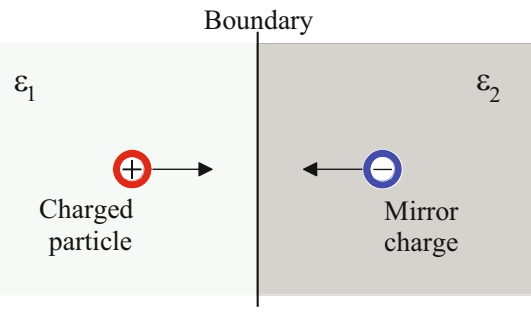

(a)

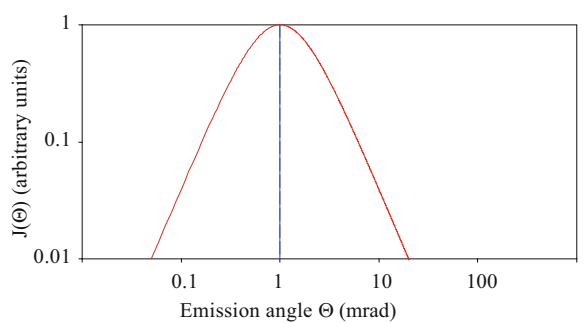

(b)

Fig. 7.29 (a) Schematic representation of the production of transition radiation at a boundary. (b) Transition radiation as function of the emission angle for $\gamma=10^{3}$. Eq. (7.40) 
direction for backward transition radiation. $N$ is the total number of emitted photons. Equation (7.40) is plotted in Fig. 7.29b.

The energy radiated from a single surface, assuming $\varepsilon_{0} \rightarrow \varepsilon$, is given by:

$$
W=\frac{1}{3} \alpha Z^{2} \omega_{p} \gamma
$$

where $\omega_{p}$ is the plasma frequency.

\subsubsection{Plasma Frequency}

The influence of the plasma frequency was shown in the saturation of the relativistic rise expressed by the Bethe-Bloch formula, Chap. 2 and [82], due to the polarisation of the medium:

$$
\frac{\delta}{2}=\ln \frac{\omega_{p}}{I}+\ln \beta \gamma-\frac{1}{2}
$$

where $I$ and $\omega_{p}$ are respectively the mean excitation energy and the plasma frequency of the medium and $\delta$ is the density correction.

The plasma frequency, $\omega_{p}$, is the natural frequency of density oscillations of free electrons and its value depends only weakly on the wavelength. Longitudinal plasma waves are resonant at $\omega_{p}$. Transverse electromagnetic waves are absorbed below $\omega_{p}$. If $\omega<\omega_{p}$, the index of refraction has an imaginary part and the electromagnetic waves are attenuated or reflected. If $\omega \gg \omega_{p}$, the index is real and a metal becomes transparent. For large $\omega$ one can write

$$
n^{2}=1-\left(\frac{\omega_{p}}{\omega}\right)^{2}
$$

The plasma frequency is given as:

$$
\omega_{p}^{2}=\frac{N Z e^{2}}{\varepsilon_{0} m}
$$

and depends only on the total number, $N Z$, of free electrons per unit volume. The plasma frequency can be approximated with:

$$
\omega_{p}(\mathrm{eV}) \simeq 28.8 \sqrt{\frac{\rho\left(\mathrm{g} / \mathrm{cm}^{3}\right) \cdot z}{A}}
$$

where $z$ is the effective number of free electrons per unit volume. Table 7.5 gives the corresponding calculated and measured wavelength, $\lambda_{p}$, for alkali metals. $z=1$ for alkali, group 1a, metals. The calculated plasma energies in $\mathrm{Si}, \mathrm{Ge}$ and $\mathrm{InSb}$ are 
Table 7.5 Ultraviolet transmission limits of alkali metals in nm [89]

Table 7.6 Radiator material properties [90]

\begin{tabular}{l|c|c|l|l}
\hline & \multirow{2}{*}{$A$} & $Z$ & $\lambda_{p}[\mathrm{~nm}]$ & \\
\cline { 3 - 5 } Material & & & Calculated & Measured \\
\hline $\mathrm{Li}$ & 6.939 & 3 & 155 & 155 \\
\hline $\mathrm{Na}$ & 22.99 & 11 & 209 & 210 \\
\hline $\mathrm{K}$ & 39.10 & 19 & 287 & 315 \\
\hline $\mathrm{Rb}$ & 85.47 & 37 & 322 & 340 \\
\hline $\mathrm{Cs}$ & 132.95 & 55 & 362 & - \\
\hline
\end{tabular}

\begin{tabular}{l|l|l|l|l}
\hline Material & & $\begin{array}{l}\rho \\
{\left[\mathrm{g} / \mathrm{cm}^{3}\right]}\end{array}$ & $\begin{array}{l}\omega_{p} \\
{[\mathrm{eV}]}\end{array}$ & $\begin{array}{l}\mathrm{X}_{0} \\
{[\mathrm{~cm}]}\end{array}$ \\
\hline Lithium & & 0.534 & 13.8 & 148 \\
\hline Beryllium & & 1.84 & 26.1 & 34.7 \\
\hline Aluminium & & 2.70 & 32.8 & 8.91 \\
\hline Polyethylene & $\mathrm{CH}_{2}=\mathrm{CH}_{2}$ & 0.925 & 20.9 & 49 \\
\hline Mylar & $\mathrm{C}_{5} \mathrm{H}_{4} \mathrm{O}_{2}$ & 1.38 & 24.4 & 28.7 \\
\hline Air & & $2.2 \cdot 10^{-3}$ & 0.7 & $30.9 \cdot 10^{3}$ \\
\hline & & & &
\end{tabular}

based on four valence electrons per atom. In a dielectric the plasma oscillation is physically the same as in a metal: the entire valence electron sea oscillates back and fourth with respect to the ion core. Table 7.6 tabulates properties of some commonly used radiator material.

\subsubsection{Formation Zone}

A minimum thickness is required in order to efficiently produce the transition radiation as the evanescent field has a certain extension. This is the formation zone and is illustrated in Fig. 7.30 for a stack of aluminium, $\omega_{p}(\mathrm{Al}) \sim 32.8 \mathrm{eV}$, and air, $\omega_{p}$ (air) $\sim 0.7 \mathrm{eV}$. The length of the formation zone, $d$, can be written as:

$$
d=\frac{2 c}{\omega}\left[\gamma^{-2}+\Theta^{2}+\left(\frac{\omega_{p}}{\omega}\right)^{2}\right]^{-1}
$$

which has a maximum, $d_{\max }$, at $\omega=\gamma \omega_{p} / \sqrt{2}$ for $\Theta=\gamma^{-1}$, which is equivalent to the maximum intensity as can be seen from Eq. (7.40) and Fig. 7.29b.

$$
d_{\max }(\mu \mathrm{m}) \sim 140 \cdot 10^{-3} \frac{\gamma}{\omega_{p}(\mathrm{eV})}
$$

Inserting Eq. (7.45) in Eq. (7.47), we see that for media with a density in the order of $1, \omega_{p} \simeq 20 \mathrm{eV}$ and $\mathrm{d}_{\max } \simeq 7 \mu \mathrm{m}$ for $\gamma=1000$. For a gas, $\omega_{p}$ is about 30 times smaller due to the reduced density and $d_{\max }$ thereby 30 times longer for same $\gamma$. 


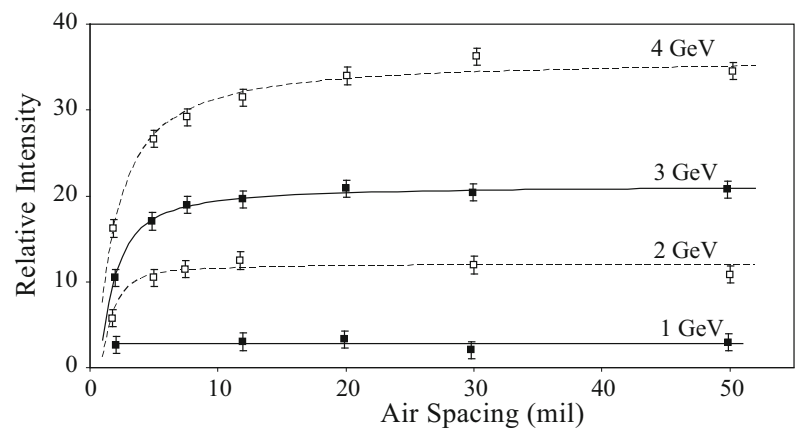

Fig. 7.30 Relative intensity of transition radiation for different air spacing. Each radiator is made of 231 aluminium foils 1 mil thick. $(1 \mathrm{mil}=25.4 \mu \mathrm{m})$. Particles used are positrons of $1-4 \mathrm{GeV}$ energy $(\gamma=2000-8000)$. Adapted from [91]

Using numbers for the experimental set-up in Fig. 7.30, we get $d_{\max } \sim 1.5 \mathrm{~mm}$ for $\gamma=8000$.

\subsubsection{Transition Radiation Detectors}

From the discussion above, transition radiation can be characterized by the following:

- Transition radiation is a prompt signal.

- Transition radiation is not a threshold phenomenon.

- The total radiated power from a single interface is proportional to $\gamma$.

- The mean emission angle is inversely proportional to $\gamma$.

In general terms, there are two different types of transition radiation detectors:

1. The detectors working in the low energy, optical, range.

2. The detectors working in the X-ray range.

We will briefly introduce the first one and use a little more space on the second class of X-ray transition radiation detectors.

\subsubsection{Optical Transition Radiation Detectors}

J.E. Lilienfeld [92] was probably the first, ${ }^{22}$ in 1919 to observe that in addition to X-rays, radiation ranging from visible light through the ultraviolet is emitted

\footnotetext{
${ }^{22}$ This statement has been contested over the years and could be due to a confusion between transition, Cherenkov radiation and bremsstrahlung. See [93].
} 


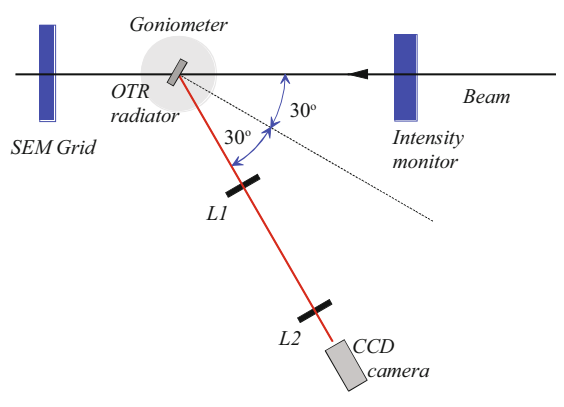

(a)

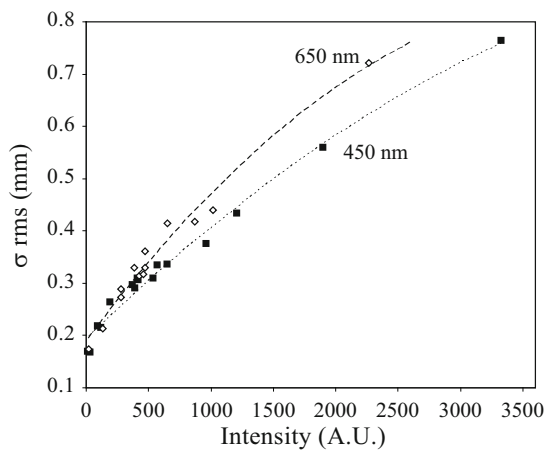

(b)

Fig. 7.31 (a) Sketch of an experimental set-up for measurement of optical transition radiation with secondary emission, SEM, grid and beam intensity monitor. The transition radiation foil is tilted by $30^{\circ}$ with respect to the beam line. The optical system is defined by two lenses and a CCD camera. (b) Measured rms beam size values as a function of the total intensity for $\lambda=450$ and $650 \mathrm{~nm}$ at $2 \mathrm{GeV}$. Adapted from [94]

when electrons approach a metallic surface. This radiation has a characteristic polarisation, spectrum and intensity. A variation to this radiation occurs when the charged particle moves roughly parallel to a conducting undulated surface. An oscillating dipole will be set up with a frequency related to the particle velocity and the undulation. The radiated power is small, but due to the microscopic source area, the brightness can be large. This has, amongst a range of other usages, found an application in accurate beam diagnostics equipments.

As an example, we will use an experiment to investigate the geometrical resolution of optical transition radiation as shown in Fig. 7.31a [94]. Integrating Eq. (7.40) over the solid angle gives:

$$
\frac{d N}{d \omega} \simeq \frac{2 \alpha}{\pi \omega} \ln \left(\gamma \Theta_{\max }\right)
$$

where $\Theta_{\max }$ is the angle of maximum emission, measured by the optical spectrometer. The number of photons emitted is small. This must be compensated by a large number of particles in the beam.

The mathematics for such a set-up is given in [95]. The diffraction, or the Heisenberg uncertainty principle in the transverse phase-space of the photon, sets the lower limit for the size of the emitting surface:

$$
\Delta b_{i} \geq \frac{\lambda}{2 \pi} \frac{1}{2 \Delta \Theta_{i}} \quad(i=x, y)
$$

where $\lambda \sim 600 \mathrm{~nm}$ is the observed wavelength. $b_{i}$ and $\Theta_{i}$ are the components of the impact parameter $\mathbf{b}$ and the photon direction. $\Delta \Theta_{i}$ and $\Delta b_{i}$ refer to rms values. Setting $\Delta \Theta=\gamma^{-1}$, or full acceptance for the photons, the resolution becomes 
Table 7.7 Parameters for the fit to the data [94] and plotted in Fig. 7.31b

\begin{tabular}{l|l|l}
\hline Parameter & $\lambda=450 \mathrm{~nm}$ & $\lambda=650 \mathrm{~nm}$ \\
\hline$\rho$ & $176 \pm 12 \mu \mathrm{m}$ & $163 \pm 25 \mu \mathrm{m}$ \\
\hline$a$ & $(9 \pm 5) \cdot 10^{-5}$ & $(6 \pm 3) \cdot 10^{-5}$ \\
\hline$b$ & $1.12 \pm 0.09$ & $1.12 \pm 0.06$ \\
\hline
\end{tabular}

proportional to $\gamma \cdot \gamma=10^{5}$ would give $\Delta b \geq 5 \mathrm{~mm}$. This effect can be limited by the introduction of an iris in the optical path as in [96].

The results from [94] are shown in Fig. 7.31b. As expected, the resolution is weakly dependent on the intensity of the beam, but the total uncertainty is small. The measurement points are fitted to $\sigma_{\mathrm{rms}}=\sqrt{\rho^{2}+a I^{b}}$, where $a$ and $b$ are fit parameters, $\rho$ is the real beam dimension and $I$ is the beam intensity. These are given in Table 7.7.

Another promising application for optical transition radiation is in aeroge $\mathrm{l}^{23}$ Cherenkov detectors [97].

\subsubsection{X-ray Transition Radiation Detectors}

Following [98], the total radiated energy from a single surface per unit of frequency, can be approximated by:

$$
\left[\frac{d W}{d \omega}\right]_{\text {s.s. }}=\frac{\alpha}{\pi}\left[\frac{1+r+2 X_{1}^{2}}{1-r} \ln \frac{X_{1}^{2}+1}{X_{1}^{2}+r}-2\right]
$$

where

$$
X_{1}=\frac{\omega}{\gamma \omega_{p 1}} \quad \text { and } \quad r=\frac{\omega_{p 2}^{2}}{\omega_{p 1}^{2}} \sim \frac{\rho_{2}}{\rho_{1}}
$$

The suffix 1 and 2 denote medium 1 and 2. $\omega_{p i}$ is the plasma frequency for medium $i . r$ will be assumed to be small and in the range of $10^{-3}$, which corresponds to a $\rho=1$ to gas interface.

By analysing Eq. (7.50), which is plotted in Fig. 7.32a, three distinct regimes can be examined:

1. If $\gamma \ll \omega / \omega_{p 1}$ then $X_{1} \gg 1$ and $d W / d \omega \sim \alpha / 6 \pi X_{1}^{4}$, which is a small number. This results in a frequency cut-off and thereby $\omega \leq \gamma \omega_{p 1}$.

2. If $\omega / \omega_{p 1} \ll \gamma \ll \omega / \omega_{p 2}$ then $d W / d \omega \propto \ln X_{1}^{-1}$. That is, the total radiated power increases logarithmically with $\gamma$.

3. If $\gamma \gg \omega / \omega_{p 2}$ then $X_{1} \ll \sqrt{r}$. Then the total radiated power is approximately constant.

${ }^{23}$ See Sect. 7.4.2.2. 


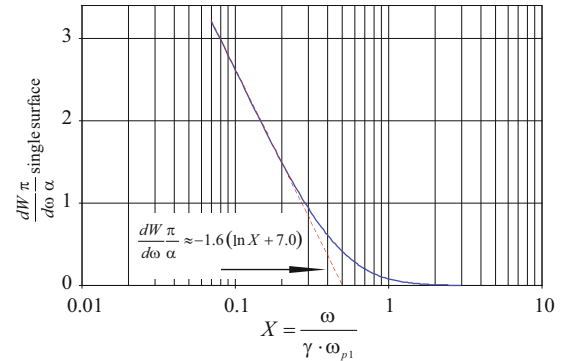

(a)

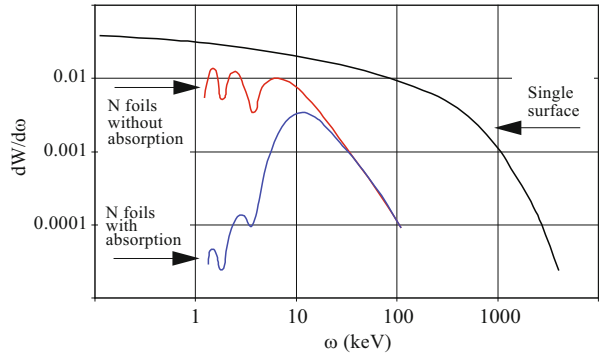

(b)

Fig. 7.32 (a) Total radiated energy from a single surface per unit of frequency as function of the dimensionless variable $X=\omega / \gamma \omega_{p 1}$. (b) Intensity of the forward radiation divided by the number of interfaces for $20 \mu \mathrm{m}$ polypropylene $\left(\omega_{p}=21 \mathrm{eV}\right)$ and $180 \mu \mathrm{m}$ helium $\left(\omega_{p}=0.27 \mathrm{eV}\right)$. Adapted from [99]

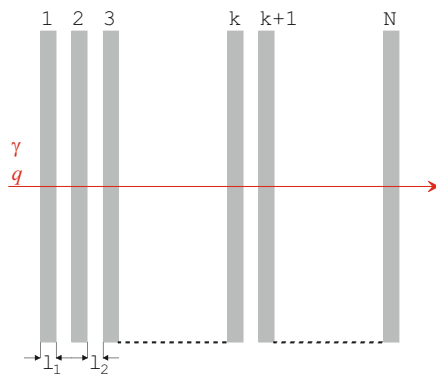

(a)

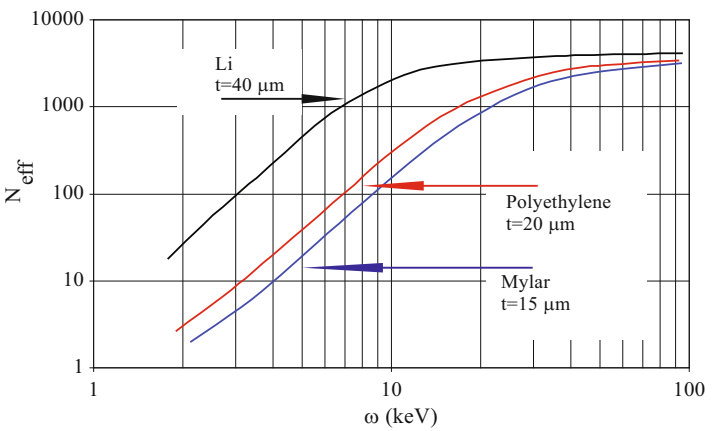

(b)

Fig. 7.33 (a) Sketch of a periodic transition radiation radiator. (b) The effective number of foils in a radiator as function of photon energy. Adapted from [90]

It can be shown that the mean radiated energy in this single surface configuration can be written as:

$$
W \simeq 2 \alpha \gamma \omega_{p 1} / 3
$$

and that the number of high energy photons produced are of the order of $\alpha$ when taking into account the frequency cut-off discussed above:

$$
N_{\text {photons }}\left(\omega>0.15 \gamma \omega_{p 1}\right) \simeq \alpha / 2
$$

A large number of interfaces are therefore required to have an effective detector with a sufficient signal-to-noise ratio. A periodic transition radiation radiator is sketched in Fig. 7.33a. It should be noted that the radiators do not need to be rigorously periodic, but it is helpful for the calculation of the yield. 
The basic mathematics can be found in $[85,90,98]$. Computational models can be found in [100]. The effective final number of transition radiation high energy photons at the end of the radiator stack is a function of constructive and destructive effects. See Fig. 7.32b. We will list the main effects here:

- The total radiated energy of a single surface is proportional to the plasma frequency and thereby proportional to $\sqrt{Z}$ of the material. Equation (7.44). The absorption of these photons is governed by photo-electric effects and the absorption coefficients in the stack. This goes approximately like $Z^{5}$. The radiator material should therefore be of low $Z$.

- The thickness of the radiator material, $I_{1}$ in Fig. 7.33a, must be large enough to contain the formation zone for the required $\gamma$, but short enough not to introduce multiple scattering effects and bremsstrahlung. The gas density will always introduce a negative effect and should be kept as low as possible.

For a practical transition radiation radiator and following [90], the expression of the total flux, is then represented by an integration over the emission angle $\Theta$ and a function which represents the incoherent addition of the single foil intensities and includes the photon absorption in the radiator. The effective number of foils in the radiator can then be expressed as:

$$
N_{\mathrm{eff}} \simeq \frac{1-\exp (-N \sigma)}{1-\exp (-\sigma)}
$$

where $\sigma=(\kappa \rho t)_{\text {foil }}+(\kappa \rho t)_{\text {gas }}$ and $\kappa, \rho$ and $t$ are respectively the absorption coefficient, density and thickness of the material. The self-absorption of the photons from transition radiation limits the yield and $N_{\text {eff }} \rightarrow 1 /[1-\exp (-\sigma)]$ for $N \rightarrow$ $\infty$. A typical mean energy for the photons in a practical radiator is in the range of $10 \mathrm{keV}$. See Fig. 7.32b. The spectrum will be softer for foils with lower plasma frequencies. Since $N_{\text {eff }}$ in Eq. (7.54) is depending on the absorption coefficient through the frequency of the photon, $N_{\text {eff }}$ will saturate for high frequencies as shown in Fig. 7.33b.

\subsubsection{X-ray Detectors}

Any detector which has a sufficiently high efficiency for X-rays of the order of $10 \mathrm{keV}$ can be used. In the design of the detector it should be noted that the number of transition radiation photons is small and produced very close to the path of the charged particle which will normally also traverse the detector. The traditional detector is a MWPC-like detector, Chap. 3, which directly follows the radiator. In order to enhance the signal-to-noise ratio and efficiently use the space as the effective number of interfaces in the radiator will saturate, a transition radiation detector is therefore normally many radiator/detector assemblies. 


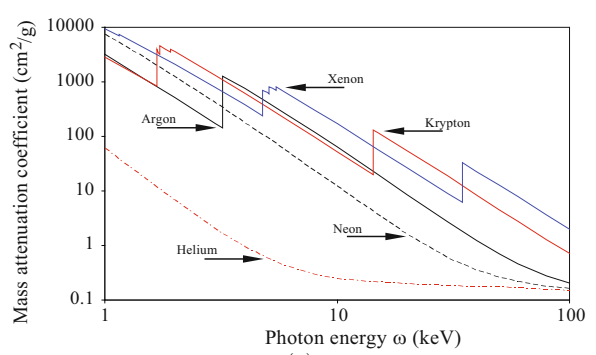

(a)

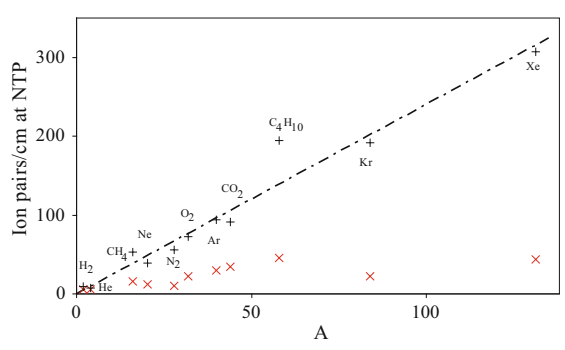

(b)

Fig. 7.34 (a) X-ray mass attenuation coefficient, $\mu / \rho$, as function of the photon energy. $\mu / \rho=$ $\sigma_{\text {tot }} / u A$, where $u=1.660 \times 10^{-24} \mathrm{~g}$ is the atomic mass unit, $A$ is the relative atomic mass of the target element and $\sigma_{t o t}$ is the total cross section for an interaction by the photon. Data from http:// physics.nist.gov/PhysRefData/. (b) The $(\times)$ primary and $(+)$ total number of ion pairs created for a minimum ionizing particle per $\mathrm{cm}$ gas at normal temperature and pressure as function of molecular mass $A$ [101]

The ionization loss, $d E / d x$, from the charged particle will create charge clusters. Some of them rather far from the track due to $\delta$-electrons. The absorption of transition radiation photons will produce a few local strong charge clusters. The choice of gas is therefore a compromise between photon absorption length, Fig. 7.34a, and the background from $d E / d x$, Fig. 7.34b. The optimal gas thickness is about one absorption length for $10 \mathrm{keV}$. Xenon is the preferred gas with a chamber thickness of $10-15 \mathrm{~mm}$. See discussion in [90]. $\mathrm{CO}_{2}$, or similar, is added as quencher.

A minimum ionizing particle, MIP, will produce a total of $\sim 310$ ion pairs per $\mathrm{cm}$ xenon gas. Figure $7.34 \mathrm{~b}$. The relativistic rise is about $75 \%$ in xenon at $1 \mathrm{~atm}$, or about 550 ion pairs $/ \mathrm{cm}$ will be produced by a high $\gamma$ charged particle. The average energy required to create an ion pair in a gas, is typically $25-35 \mathrm{eV}$. For xenon it is measured to $22.1 \pm 0.1 \mathrm{eV}$ [102], or about double the ionization energy for the least tightly bound shell electron. A $10 \mathrm{keV}$ transition radiation photon will then produce about 450 ion pairs. The signal-to-noise ratio will be further reduced due to Landaufluctuations and gain variations in the detector and electronics. Additional background might arise from curling in a magnetic field, bremsstrahlung and particle conversions. The challenge is then to correctly identify the photon cluster from a $d E / d x$ signal of about the same strength. We will illustrate this by looking more closely at the choices made by the ALICE [103] and ATLAS [104] experiments.

\subsubsection{ATLAS Transition Radiation Tracker}

In the ATLAS experiment, the transition radiation tracker (TRT) in the barrel comprises many layers of gaseous straw tube elements interleaved with transition radiation material. Figure 7.35. With an average of 36 hits per track, it provides continuous tracking to enhance the pattern recognition and improve the momentum 


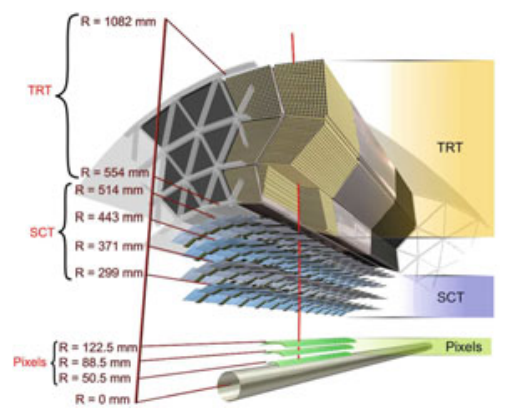

(a)

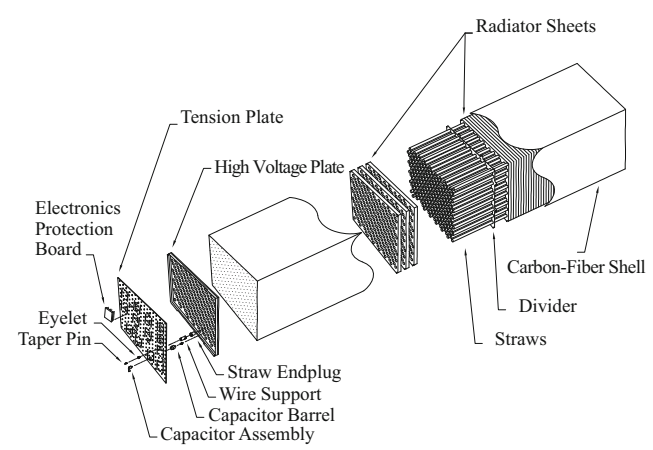

(b)

Fig. 7.35 (a) ATLAS Detector. Drawing showing the sensors and structural elements traversed by a charged track of $10 \mathrm{GeV} p_{t}$ in the barrel inner detector (pseudo rapidity $\eta=0.3$ ). The track traverses approximately 36 axial straws of $4 \mathrm{~mm}$ diameter contained in the barrel transitionradiation tracker modules. [104]. (b) Layout of an ATLAS Barrel TRT module. The ATLAS TRT collaboration et al. [105] with permission

resolution over $|\eta|<2.0^{24}$ and electron identification complementary to that of the calorimeter over a wide range of energies. A similar detector is placed in the forward direction.

The transition radiator material which completely surrounds the straws inside each module, Fig. $7.35 \mathrm{~b}$, consists of polypropylene-polyethylene fibre mat about $3 \mathrm{~mm}$ thick. The fibres are typically $19 \mu \mathrm{m}$ in diameter and are formed from polyethylene clad polypropylene material. The fibres are formed into fabric plies with $3 \mathrm{~mm}$ thickness and a density of about $0.06 \mathrm{~g} / \mathrm{cm}^{3}$. The absorption length for the lowest energy photons of interest $(5 \mathrm{keV})$ is about $17 \mathrm{~mm}$ in the radiator material.

The ATLAS TRT uses two thresholds to discriminate between digitisations from tracks and those from transition radiation:

1. Low threshold, LT, for tracking which is set to $\sim 300 \mathrm{eV}$ with 8 digitisations over $25 \mathrm{~ns}$.

2. High threshold, HT, set in the range 5-7 keV with 1 digitisation over $25 \mathrm{~ns}$ and read out in $75 \mathrm{~ns}$ segments.

As the $\beta \gamma$ of the traversing particles will vary greatly, and thereby the ionization in the straw tubes, a Time-over-Threshold parameter can be defined from the LT digitisations in order to enhance the signal-to-noise estimate for the transition radiation signal.

Particle identification properties of the TRT Barrel using transition radiation were studied at several different beam energies. The good agreement between $2 \mathrm{GeV}$ low

\footnotetext{
${ }^{24}$ Pseudo rapidity, $\eta$, is describing the angle of a particle relative to the beam axis. $\eta=$ $-\ln \left[\tan \left(\frac{\Theta}{2}\right)\right]=\frac{1}{2} \ln \left[\frac{|\mathbf{p}|+p_{L}}{|\mathbf{p}|-p_{L}}\right] . \Theta$ is the angle between the particle momentum and the beam axis, $\mathbf{p}$ is the momentum vector and $p_{L}$ is the longitudinal momentum component.
} 


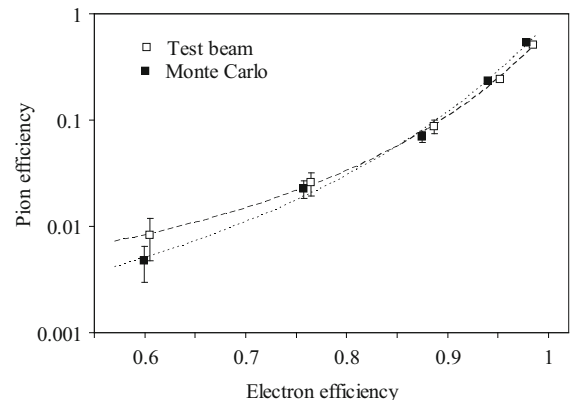

(a)

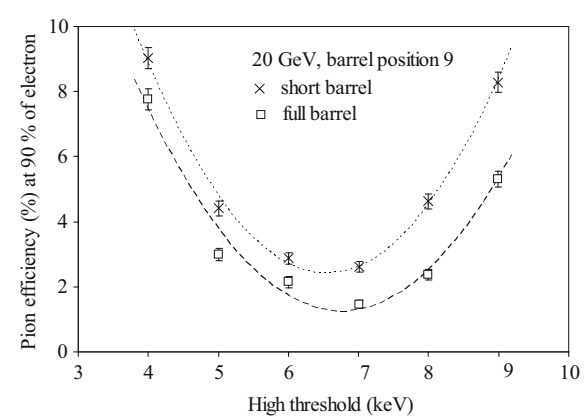

(b)

Fig. 7.36 (a) ATLAS TRT test beam. Pion rejection curve for a $2 \mathrm{GeV}$ e/ $\pi$ beam. Cornelissen and Liebig [106] with permission. (b) ATLAS TRT test beam. e/ $\pi$ rejection power as a function of the high level threshold. Full barrel: all barrel straw layers are active. Short barrel: particle crosses the barrel in the central area where the first 9 layers do not have active anode wires. The ATLAS TRT collaboration et al. [105] with permission

energy data and simulation is shown in Fig. 7.36a. The results for $20 \mathrm{GeV}$ beam energy are shown in Fig. 7.36b. On this figure the pion rejection power is shown as a function of the high level threshold at two beam positions along the straw. The upper points are when beam particles crossed the Barrel module $40 \mathrm{~cm}$ from its edge. At this position the first 9 straw layers are not active. The lower points are when the beam is positioned $20 \mathrm{~cm}$ from the edge of the Barrel where all 73 straw layers are active. As seen in this figure the best particle identification properties for the TRT Barrel are at a threshold of about $7 \mathrm{keV}$. Pion mis-identification in that case is $1.5-3 \%$ at $90 \%$ of the electron efficiency.

\subsubsection{ALICE Transition Radiation Detector}

The main purpose of the ALICE Transition Radiation Detector (TRD) [103, 107] is to provide electron identification in the central barrel for momenta above $1 \mathrm{GeV} / c$. Below this momentum electrons can be identified via specific energy loss measurement in the TPC. Above $1 \mathrm{GeV} / c$ transition radiation from electrons passing a radiator can be exploited together with the specific energy loss in a suitable gas mixture to obtain the necessary pion rejection capability. The chamber geometry and the read-out electronics were chosen to reconstruct track segments. Since the angle of the track segment with respect to the origin is a measure of the transverse momentum of the electron, this information is used in the first level trigger within $5 \mu \mathrm{s}$ of the collision.

The pion rejection is governed by the signal-to-background ratio in the measurement of $\mathrm{J} / \Psi$ production and its $p_{t}$ dependence. This led to the design goal for the pion rejection capability of a factor 100 for momenta above $1 \mathrm{GeV} / c$ in central Pb$\mathrm{Pb}$ collisions. 


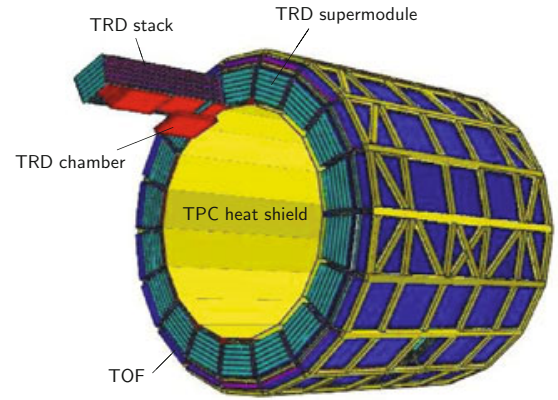

(a)

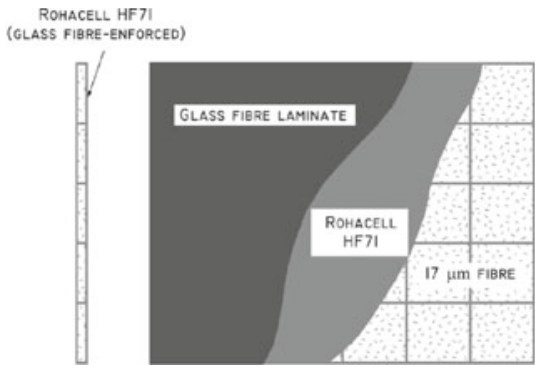

(b)

Fig. 7.37 (a) Schematic drawing of the TRD layout in the ALICE space frame. Shown are 18 super modules each containing 30 readout chambers (red) arranged in five stacks of six layers. One chamber has been displaced for clarity. On the outside the TRD is surrounded by the TimeOf-Flight (TOF) system (dark blue). On the inside the heat shield (yellow) towards the TPC is shown. The ALICE Collaboration et al. [103] with permission. (b) The principle design of the TRD sandwich radiator. The ALICE Collaboration et al. [107] with permission

The TRD consists of 540 individual readout detector modules. Figure 7.37a. Each detector element consists of a carbon fibre laminated Rohacell ${ }^{25} /$ polypropylene fibre sandwich radiator, Fig. $7.37 \mathrm{~b}$, of $48 \mathrm{~mm}$ thickness, a drift section of $30 \mathrm{~mm}$ thickness, or about $2 \mu \mathrm{s}$, and a multi-wire proportional chamber section $(7 \mathrm{~mm})$ with pad readout.

Following [108], employing the drift time information in a bidimensional likelihood [109], the pion rejection capability can be improved by about $60 \%$ [110] compared to the standard likelihood method on total deposited charge. This method is the simplest way of extending the standard method. However, it does not exploit all recorded information, namely the amplitude of the signal in each time bin. Along a single particle track this information is highly correlated, Fig. 7.38a, due to

- the intrinsic detector signal, in particular since a Xe-based mixture is used

- the response of the front-end electronics used to amplify the signals.

Under these circumstances, the usage of a neural network (NN) algorithm is a natural choice for the analysis of the data. The result of the data analysis from a $2-6 \mathrm{GeV} / c$ mixed e/ $\pi$ test beam is shown in Fig. 7.38b [108]. Neural Network algorithm might improve the pion rejection significantly by a factor larger than 3 for a momentum of $2 \mathrm{GeV} / c$ compared to other methods.

The detector was completed in the LS 1 before RUN 2 at LHC. Since then it provides coverage of the full azimuthal acceptance of the central barrel. Figure 7.39 shows the $p_{T}$ spectra of electron candidates with 6 layers identified using the TPC and the TOF in the minimum-bias and triggered data sample. The expected onset

\footnotetext{
${ }^{25}$ ROHACELL is a close cell polymethacrylimide- (PMI-) rigid foam by Evonik Industries AG, Germany.
} 


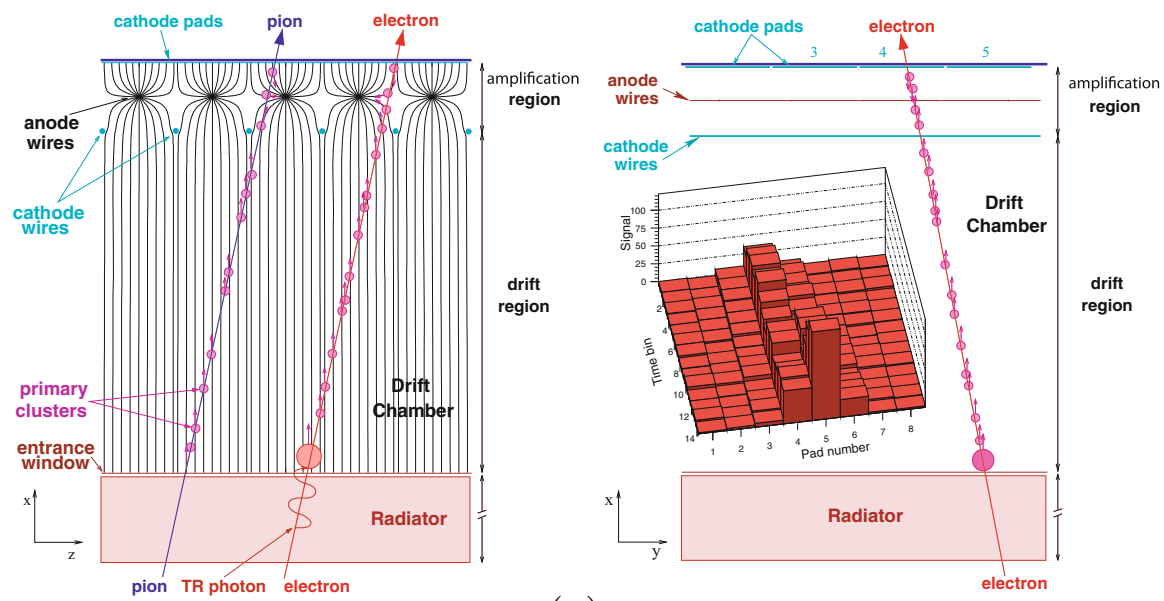

(a)

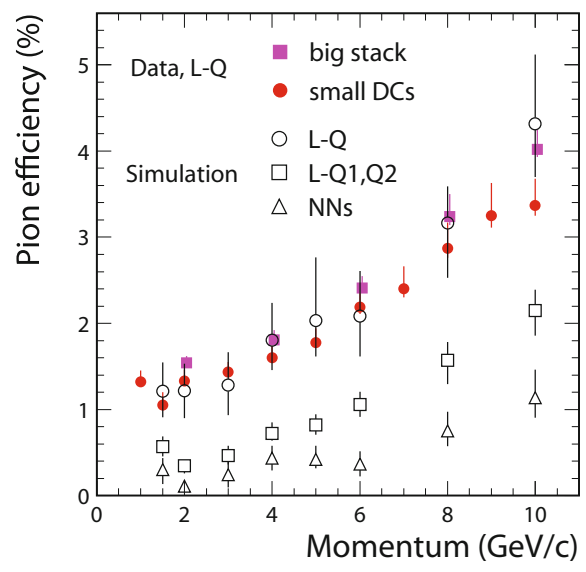

(b)

Fig. 7.38 (a) Schematic cross-sectional view of an ALICE detector module in $r z$ and $r \phi$ direction. The inset shows the charge deposit from an inclined track which is used for momentum reconstruction. The ALICE Collaboration et al. [103] with permission. (b) Measured pion efficiency as a function of beam momentum applying likelihood on total deposited charge (LQ) (full symbols) measured with a stack of six chambers and smaller test chambers. Results are compared to simulations (open symbols) for $90 \%$ electron efficiency and six layers. These simulations were extended to two-dimensional likelihood on deposited charge and position (LQ1, Q2) and neural networks (NN). The ALICE Collaboration et al. [103] with permission

at the trigger threshold of $3 \mathrm{GeV} / c$ is observed for the triggered events and shows in comparison to the corresponding spectrum from minimum-bias collisions an enhancement of about 700 . At $90 \%$ electron efficiency, a pion rejection factor of 


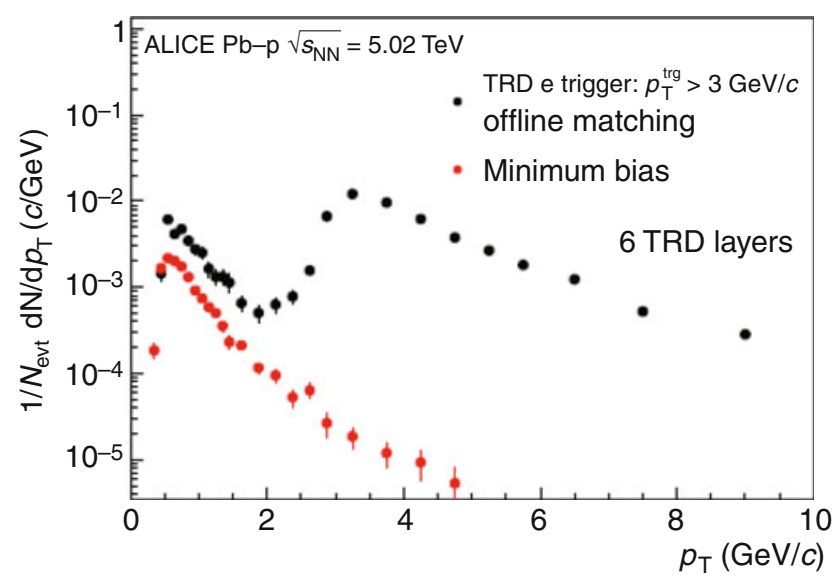

Fig. 7.39 $p_{T}$ spectra of identified electrons for the minimum-bias and TRD-triggered data sample of $\mathrm{Pb}$-p collisions at $\sqrt{s}_{N N}=5.02 \mathrm{TeV}$. For the result of the TRD-triggered sample, electrons from photon conversions in the detector material were rejected by matching the online track with a track in the TPC. Reference [111]

about 70 is achieved at a momentum of $1 \mathrm{GeV} / c$ for simple identification algorithms. When using the temporal evolution of the signal, a pion rejection factor of up to 410 is obtained.

\section{References}

1. B. Dolgoshein, Complementary particle ID: transition radiation and $\mathrm{d} E / \mathrm{d} x$ relativistic rise, Nucl. Instrum. Methods Phys. Res., A: 433 (1999) 533

2. C. Ward et al., A Large Aperture Time-Of-Flight Counter System, Nucl. Instr. and Meth. 30(1964)61-68

3. G. Charpak, L. Dick and L. Feuvrais, Location of the position of a particle trajectory in a scintillator, Nucl. Instr. and Meth. 15(1962)323-326

4. W. Klempt, Review of particle identification by time of flight techniques, Nucl. Instrum. Methods Phys. Res., A: 433 (1999) 542

5. A. Rabl and R. Winston, Ideal concentrators for finite sources and restricted exit angles, Applied Optics, Vol. 15 Issue 11, pp.2880-2883 (1976)

R.H. Hildebrand and R. Winston, Throughput of diffraction-limited field optics systems for infrared and millimetric telescopes: erratum, Applied Optics, Vol. 21 Issue 17, pp.3065-3065 (1982)

6. W. Blaschke and G. Thomsen, Vorlesungen Uber Differential-Geometrie und Geometrische Grundlagen von Einsteins Relativitatstheorie. I: Elementaire Differentialgeometrie, Dritte Erweiterte Auflage. Bearbeitet under herausgegeben, DOVER PUBLICATIONS (1945)

7. T. Kobayashi and T. Sugitate, Test of Prototypes for a Highly Segmented TOF Hodoscope, Nucl. Instrum. Methods Phys. Res., A: 287 (1990) 389-396

8. C. Field et al., Development of photon detectors for a fast focusing DIRC, Nucl. Instrum. Methods Phys. Res., A 553 (2005) 96-106 
9. J. Blazej et al., Gallium-based avalanche photodiode optical crosstalk, Nucl. Instrum. Methods Phys. Res., A: 567 (2006) 239-241, and references therein.

10. J. Va'vra et al., A 30 ps timing resolution for single photons with multi-pixel Burle MCPPMT, Nucl. Instrum. Methods Phys. Res., A: 572 (2007) 459-462

11. A.N. Akindinov et al., Latest results on the performance of the multigap resistive plate chamber used in the ALICE TOF, Nucl. Instrum. Methods Phys. Res., A: 533 (2004) 74 78

12. J. Va'vra, PID techniques: Alternatives to RICH methodes, Nuclear Instruments \& Methods in Physics Research A (2017), http://dx.doi.org/10.1016/j.nima.2017.02.075

13. A. Bravar, Recent Results from NA61 (Flux Related Systematic Uncertainties) and Recent Results from T2K (Overall Systematic Uncertainties), http://www.t2k.org/docs/talk/225, 2015-11-06

N. Abgrall et al., Measurements of cross sections and charged pion spectra in proton-carbon interactions at $31 \mathrm{GeV} / \mathrm{c}$, PHYSICAL REVIEW C 84, 034604 (2011)

14. S. Afanasiev et al., The NA49 Large Acceptance Hadron Detector, CERN-EP/99-001, 6. January 1999

15. P.A. Cherenkov, Visible glow under exposure of gamma irradiation, Dokl. Acad. Nauk. USSR 2(1934)451

S.I. Vavilov, About possible reasons of blue gamma-glow of liquids, Dokl. Acad. Nauk. USSR 2(1934)457

I.M. Frank and I.E. Tamm, Coherent Radiation of Fast Electrons in a Medium, Dokl. Acad. Nauk. USSR 14(1937)107

16. J.V. Jelley, Čerenkov Radiation and its Application, London: Pergamon Press, 1958 V.P. Zrelov, Čerenkov Radiation in High Energy Physics, Jerusalem, Israel Program for Scientific Translations, 1970

17. J. Séguinot, Les Compteurs Cherenkov: Applications et Limites pour l'Identification des Particules. Développements et Perspectives, CERN-EP/89-92, LPC/89-25, 31 juillet 1989

18. J.D. Jackson, Classical Electrodynamics, John Wiley \& Sons, Inc.,1998, ISBN 0-471-30932-X

19. W.W.M. Allison and P.R.S. Wright, The Physics of Charged Particle Identification, in:

T. Ferbel (ed.), Experimental Techniques in High-Energy Nuclear and Particle Physics, ISBN 981-02-0867-7, World Scientific Publishing Company, Inc. 1999.

20. Landolt-Börnstein, Eigenschaften der Materie in ihren Aggregatzuständen, 8. Teil Opische Konstanten.

21. C.C. Chen and W.A. Steel, Molecular calculation of the dielectric constant of $\mathrm{N}_{2}$ and $\mathrm{CO}_{2}, \mathrm{~J}$. Chem. Phys., 75(1)383-387

22. DuPont, Freon Technical Bulletin B-32, 32A

23. P.W. Langhoff and M. Karplus, Pade Summation of the Cauchy Equation, Journal of the Optical Society of America, 59(1969)863

24. J. Koch, Nova acta Soc. Upsal. (4) Nr. 5, 1909

C. u. M. Cuthbertson, Proc. Roy. Soc. London (A) 83, 151, 1910

25. E. Albrecht et al., VUV absorbing vapours in n-perfluorocarbons, Nucl. Instr. and Meth. in Phys. Res. A 510(2003)262-272

26. D.E. Gray (ed.), American Institute of Physics handbook, McGraw-Hill Book Company. Inc., 1957

27. E. Efimov and S. Stone, A novel LiF radiator for RICH detectors, Nucl. Instr. and Meth. in Phys. Res. A 371(1996)79-81

28. B. Dey et al., Design and performance of the focusing DIRC detector, Nuclear Instruments and Methods in Physics Research A 775(2015)112-131

J. Schwiening, The DIRC detector at the SLAC B-factory PEP-II: operational experience and performance for physics application, Nucl. Instr. and Meth. in Phys. Res. A 502(2003)67-75 P. Coyle et al., The DIRC counter: a new type of particle identification device for B factories, Nucl. Instr. and Meth. in Phys. Res. A 343(1994)292-299 
29. Blair N. Ratcliff, Imaging rings in Ring Imaging Cherenkov counters, Nucl. Instr. and Meth. in Phys. Res. A 502(2003)211-221

30. J. Fast, The Belle II imaging Time-of-Propagation (iTOP) detector, Nuclear Instruments \& Methods in Physics Research A (2017), http://dx.doi.org/10.1016/j.nima.2017.02.045

Y. Enari et al., Progress report on Time-Of-Propagation counter-a new type of ring imaging Cherenkov detector, Nucl. Instr. and Meth. in Phys. Res. A 494(2002)430-435

31. G. S. Varner, Waveform-sampling electronics for Cherenkov detectors, https://indico.cern.ch/ event/393078/contributions/2241768/attachments/1334816/2007370/1-WaveformReadout_ Varner_RICH2016.pdf

32. M.J. Charles and R. Forty, TORCH: Time of flight identification with Cherenkov radiation, Nuclear Instruments and Methods in Physics Research A 639(2011)173?176

T. Gys et al., The TORCH detector R \& D: Status and perspectives, Nuclear Instruments and Methods in Physics Research A (2017), http://dx.doi.org/10.1016/j.nima.2017.02.060

33. C. Schwarz et al., The PANDA DIRC Detectors at FAIR, arXiv:1707.09269 [physics.ins-det]

34. M. Cantin et al., Silica aerogels used as Cherenkov radiators, Nucl. Instr. and Meth. 118(1974)177-182

35. H. Gordon et al., The Axial Field Spectrometer at the CERN ISR, CERN-EP/81-34

36. L. A. Paquette (ed), D. Crich (ed), Ph. L. Fuchs (ed) and G. Molander(ed), Encyclopedia of Reagents for Organic Synthesis, Wiley; 2 edition (May 18, 2009)

L. Rösch, P. John and R. Reitmeier, Silicon Compounds, Organic, Ullmann's Encyclopedia of Industrial Chemistry, Wiley-VCH; Sixth edition (February 21, 2003)

37. T. Bellunato et al., Refractive index of aerogel: uniformity and dispersion law, Nucl. Instr. and Meth. in Phys. Res. A 595(2008)183-186

T. Bellunato et al., Refractive index dispersion law of silica aerogel, Eur. Phys. J. C52(2007)759-764

38. D. Perego. Private communication, March 2008

39. A.Yu. Barnyakov et al., Focusing Aerogel RICH optimization, Nucl. Instr. and Meth. in Phys. Res. A 595(2008)100-103

40. M.Born and E. Wolf, Principles of Optics, University Press, Oxford, ISBN 0-521-64222-1

41. R. Pestotnik et al., The aerogel Ring Imaging Cherenkov system at the Belle II spectrometer, Nuclear Instruments \& Methods in Physics Research A (2017), http://dx.doi.org/10.1016/j. nima.2017.04.043

42. O. Chamberlain, E. Segre, C. Wiegand and Th. Ypsilantis, Observation of antiprotons, Phys. Rev. 100, No. 3, 947 (1955).

43. J. Litt and R. Meunier, Čerenkov counter technique in high-energy physics, Annu. Rev. Nucl. Part. Sci.: 23(1973)1-43

R. Meunier, Čerenkov Detectors, 1973 International Conference on Instrumentation for High Energy Physics, Frascati, Italy, May 8-12, 1973, Published by Laboratori Nazionali del CNEN

44. M. Bourquin et al., Particle and antiparticle production by $200 \mathrm{GeV} / \mathrm{c}$ protons in the charged hyperon beam at the CERN SPS, Nucl. Phys. B 153(1979)13-38

45. J. Séguinot and T. Ypsilantis, Photoionization and Cherenkov Ring Imaging, Nucl. Instr. and Meth., 142(1977)377; for an extensive list of RICH references, see http://alice-hmpid.web.cern.ch/alice-hmpid/basic_references.htm

46. J. Séguinot and T. Ypsilantis, A historical survey of ring imaging Cherenkov counters, Nucl. Instr. and Meth. in Phys. Res. A, 343(1994)1-29

J. Séguinot and T. Ypsilantis, Theory of ring imaging Cherenkov counters, Nucl. Instr. and Meth. in Phys. Res. A, 343(1994)30-51

47. Carl A. Heller and Aaron N. Fletcher, Oxidation and Chemiluminescence of Tetrakis (dimethylamino) ethylene. I. Reversible Reactions of Oxygen with Tetrakis (dimethylamino) ethylene and n-Decane, J. Phys. Chem.; 1965; 69(10); 3313-3317

J.P. Paris, Chemiluminescence of tetrakis-(dimethylamino)-ethylene, Photochemistry and Photobiology, 4(6)(1965)1059-1065

Sidney Toby, Paul A. Astheimer and Frina S. Toby, Chemiluminescence in the gas phase 
reaction between tetrakis-(dimethylamino)-ethylene and oxygen, Journal of Photochemistry and Photobiology A: Chemistry 67(1992)1-12

W.R. Carpenter and E. M. Bens, Influence of oxidation products on the chemiluminescent oxidation of tetrakis-(dimethyl amino)-ethylene. Naval ordnance test station China Lake CA, Report 0526608, FEB 1967

W.P. Norris, Reactions of Tetrakis-(Dimethylamino)-Ethylene with Weak Acids. Naval ordnance test station China Lake CA, Report 0111409, OCT 1972

48. D. F. Anderson, A xenon gas scintillation proportional counter coupled to a photoionization detector, Nucl. Instr. and Meth., 178(1980)125-130

Wiliam H. -M. Ku, Charles J. Hailey and Michael H. Vartanian, Properties of an imaging gas scintillation proportional counter, Nucl. Instr. and Meth., 196(1982)63-67

D. F. Anderson, Measurement of TMAE and TEA vapor pressures, Nucl. Instr. and Meth. in Phys. Res. A, 270(1988)416-418

Richard A. Holroyd et al., Measurement of the absorption length and absolute quantum efficiency of TMAE and TEA from threshold to $120 \mathrm{~nm}$, Nucl. Instr. and Meth. in Phys. Res. A, 261(1987)440-444

49. H.W. Siebert et al., The Omega RICH, Nucl. Instr. and Meth. in Phys. Res. A, 343(1994)6067

50. D. Aston et al., Development and construction of the SLD Cherenkov ring-imaging detector, Nucl. Instr. and Meth. in Phys. Res. A, 283(1989)582-589

51. E. Albrecht et al., Operation, optimisation, and performance of the DELPHI RICH detectors, Nucl. Instr. and Meth. in Phys. Res. A, 433(1999)47-58

52. M. Starič, A. Stanovnik and P. Kriẑan, Tests of a solid CsI photocathode in a thin multiwire proportional chamber, Nucl. Instr. and Meth. in Phys. Res. A, 307(1991)145-148

J. Almeida et al., Development of large area fast-RICH prototypes with pad readout and solid photocathodes, Nucl. Instr. and Meth. in Phys. Res. A. 348(1994)216-222

53. J.R. Hardy and A.M. Karo, The Lattice Dynamics and statics of alkali halide crystals, Plenum Press 1979, ISBN 0-306-4022-1

I.P. Csorba, Imaging Tubes, Howard W, Sams \& Co., Inc. 1985, ISBN 0-672-22023-7

54. C. Lu and K. T. McDonald, Properties of reflective and semitransparent CsI photocathodes, Nucl. Instr. and Meth. in Phys. Res. A. 343(1994)135-151

A. Braem et al., Technology of photocathode production, Nucl. Instr. and Meth. in Phys. Res. A, 502(2003)205-210

55. T. Meinschad, L. Ropelewski and F. Sauli, GEM-based photon detector for RICH applications, Nucl. Instr. and Meth. in Phys. Res. A. 535(2004)324-329

A. Breskin et al., Recent advances in gaseous imaging photomultipliers, Nucl. Instr. and Meth. in Phys. Res. A. 513(2003)250-255

J.M. Maia et al., Single-UV-photon 2-D imaging with multi-GEM detectors, Nucl. Instr. and Meth. in Phys. Res. A. 580(2007)373-376

L. Periale et al., Detection of the primary scintillation light from dense $\mathrm{Ar}, \mathrm{Kr}$ and $\mathrm{Xe}$ with novel photosensitive gaseous detectors, Nucl. Instr. and Meth. in Phys. Res. A. 478(2002)377383 , and references therein.

56. F. Tessarotto, Status and perspectives of gaseous photon detectors, Nuclear Instruments \& Methods in Physics Research A (2017), http://dx.doi.org/10.1016/j.nima.2017.023.011

57. I. Ariño et al., The HERA-B RICH, Nucl. Instr. and Meth. A 453 (2000) 289-295

58. P. Abbon et al., Read-out electronics for fast photon detection with COMPASS RICH-1, Nucl. Instr. and Meth. A 587 (2008) 371-387

59. M. Moritz et al., Performance Study of New Pixel Hybrid Photon Detector Prototypes for the LHCb RICH Counters, IEEE TRANSACTIONS ON NUCLEAR SCIENCE, 51(2004)1060 1066

60. LHCb Collaboration, LHCb RICH, Technical Design Report, CERN/LHCC/2000-0037, 7 September 2000

61. A. Braem et al., Development, fabrication and test of a highly segmented hybrid photodiode, Nucl. Instr. and Meth. in Phys. Res. A. 478(2002)400-403 
62. T. Gys, Micro-channel plates and vacuum detectors, Nuclear Instruments \& Methods in Physics Research A 787(2015)254-260

T. Iijima, Status and perspectives of vacuum-based photon detectors, Nuclear Instruments \& Methods in Physics Research A 639(2011)137-143

T. Gys, Status and perspectives of vacuum-based photon detectors for single photon detection, Nuclear Instruments \& Methods in Physics Research A 595(2008)136-141

63. ALICE Collaboration, ALICE Technical Design Report, Detector for High Momentum PID, CERN/LHCC 98-19, ALICE TDR 1, 14 August 1998.

64. J. Holder et al., The first VERITAS telescope, Astroparticle Physics 25 (2006) 391-401

65. P. Baillon et al., An improved method for manufacturing accurate and cheap glass parabolic mirrors, Nucl. Instrum. Methods Phys. Res., A 276(1989)492-495

P. Majumdar et al., Angular Resolution of the Pachmarhi Array of Cerenkov, Telescopes Astropart. Phys. 18(2003)333-349

66. M. Laub, Development of opto-mechanical tools and procedures for the new generation of RICH-detectors at CERN, CERN-THESIS-2006-028; LHCb-2001-130; CERN-LHCb-2001130, Prague: Prague TU, 2001

67. E. Albrecht et al., The mirror system of COMPASS RICH-1, Nucl. Instrum. Methods Phys. Res., A 502 (2003) 236-240

68. G.J. Barber et al., Glass-coated beryllium mirrors for the LHCb RICH1 detector, Nucl. Instrum. Methods Phys. Res., A 570(2007)565-57

69. J. Friese, R. Gernhäuser, P. Maier-Komor and S. Winkler, A new carbon based VUV mirror of high radiation length for the HADES RICH, Nucl. Instrum. Methods Phys. Res., A 502(2003)241-245

F.C.D. Metlica and On behalf of the LHCb Collaboration, Development of light-weight spherical mirrors for RICH detectors, Nucl. Instrum. Methods Phys. Res., A 595(2008)197199

70. T. Bellunato et al., Light composite mirrors for RICH detectors: production, characterisation and stability tests, Nucl. Instrum. Methods Phys. Res., A 538(2005)458-464

71. C. Bigongiari et al., The MAGIC telescope reflecting surface, Nucl. Instrum. Methods Phys. Res., A 518(2004)193-194

72. U. Müller et al., The Omega RICH in the CERN hyperon beam experiment, Nucl. Instrum. Methods Phys. Res., A 433(1999)71-76

73. A. Braem, C. David and C. Joram, Metal multi-dielectric mirror coatings for Cherenkov detectors, Nucl. Instrum. Methods Phys. Res., A 553(2005)182-186

74. D. Malacara (Ed.), Optical Shop Testing (Wiley Series in Pure and Applied Optics), WileyInterscience, 2007, ISBN: 978-1574446821

D. Malacara, M. Servín and Z. Malacara, Interferogram Analysis For Optical Testing, Second Edition (Optical Engineering), CRC; 2005, ISBN: 978-0471484042

75. The LHCb Collaboration, A. Augusto Alves Jr. et al, The LHCb Detector at the LHC, JINST 3 (2008) S08005.

76. G. Wilkinson, In search of the rings: Approaches to Cherenkov ring finding and reconstruction in high-energy physics, Nucl. Instr. and Meth. A 595 (2008) 228.

77. M. Adinolfi et al., Performance of the LHCb RICH detector at the LHC, Eur. Phys. J. C73 (2013) 2431, arXiv:1211.6759.

78. R. Forty and O. Schneider, RICH Pattern Recognition, LHCb note LHCb-98-040.

79. C. Buszello, LHCb RICH pattern recognition and particle identification performance, Nucl. Instr. and Meth. A 595 (2008) 245.

80. M. Starič, Track based maximum likelihood ring search, Nucl. Instr. and Meth. A 595 (2008) 237

81. LHCb collaboration: R. Aaij et al., Measurement of b-hadron branching fractions for twobody decays into charmless charged hadrons, JHEP10(2012)037

82. E. Fermi, The Ionization Loss of Energy in Gases and in Condensed materials, Phys. Rev. 57(1940)485-493 
83. V.L. Ginzburg and I.M. Frank, Radiation of a uniformly moving electron due to its transition from one medium into another, Zh. Eksp. Teor. Fiz. 16 (1946)15.

a shortened (English) version J. Phys. USSR 9 (1945), 353.

84. C.W. Fabjan and W. Struczinski, Coherent Emission of Transition Radiation in Periodic Radiators, Physics Letters 57B(1975)483-486

85. C. Leroy and P-G. Rancoita, Principles of radiation interaction in matter and detection, World Scientific, 2004, ISBN: 978-9812389091

86. M.L.Ter-Mikaelian, High-Energy Electromagnetic Processes in Condensed Media, John Wiley \& Sons, Inc, 1972, ISBN 0-471-85190-6

87. G.N. Afanasiev, V.G. Kartavenko and Yu.P. Stepanovsky, Vavilov-Cherenkov and transition radiations on the dielectric and metallic spheres, Journal of Mathematical Physics, 44(2003)4026-4056

88. V.L. Ginzburg and V.N. Tsytovich, On the Derivation of the Transition Radiation Intensity, Physics Letters 79A(1980)16-18

V.L. Ginzburg, On the radiation from uniformly moving sources, Nucl. Instrum. Methods Phys. Res., A: 248 (1986) 13-16

89. Charles Kittel, Introduction to solid state physics. - 7th ed. / New York, NY: Wiley, 1996

90. B. Dolgoshein, Transition radiation detectors, Nucl. Instrum. Methods Phys. Res., A: 326 (1993) 434-469

91. L.C.L. Yuan, C.L. Wang and H. Uto, Formation-Zone effects in transition radiation due to ultrarelativistic particles, Phys. Rev. Lett. 25 (1970) 1513-1515

92. J.E. Lilienfeld, Die sichtbare Strahlung des Brennecks von Röntgenröhren, Physik Zeitschrift, XX(12) 280, 1919

Mario Rabinowitz, Lilienfeld Transition Radiation Brought to Light, physics/0307047 (July 2003)

93. H. Boersch, C. Radeloff and G. Sauerbrey, Experimental Detection of Transition Radiation, Phys. Rev. Lett. 7(1961)52-54

94. X. Artru et al., Experimental investigations on geometrical resolution of optical transition radiation (OTR), Nucl. Instrum. Methods Phys. Res., A: 410 (1998) 148-158

95. J. Ruẑiẑka and J. Mehes, Properties of optical transition radiation for charged particle inclined flight through a finite thick plate IX, Nucl. Instrum. Methods Phys. Res., A: 250 (1986) 491502, and references therein.

96. S.D. Borovkov et al., On studying the possibility to use optical transition radiation for proton beam diagnostics, Nucl. Instrum. Methods Phys. Res., A: 294 (1989) 101-107

97. J. Ruzicka et al., On optical transition radiation of charged particles in $\mathrm{SiO}_{2}$-aerogels, Nucl. Instrum. Methods Phys. Res., A: 384 (1997) 387-402

98. X. Artru, G.B. Yodh and G. Mennessier, Practical theory of the multilayered transition radiation detector, Phys. Rev. D 16(1975)1289-1306

99. L. Fayard, Transition radiation, in: Instrumentation en Physique Nucléaire et en Pysique des Particules, les éditions de physiques, 1988, 327-340

100. J. Apostolakis et al., Parameterization models for X-ray transition radiation in the GEANT4 package, Comput. Phys. Commun. 132, 3 (2000) 241-50

V.M. Grichine and S.S. Sadilov, GEANT4 X-ray transition radiation package, Nucl. Instrum. Methods Phys. Res., A: 253 (2006) 299-302

101. F. Sauli, Principles of Operation of Multiwire Proportional and Drift Chambers, CERN 77-09

102. P. Cwetanski, Straw performance studies and quality assurance for the ATLAS transition radiation tracker, Ph.D. Thesis, Helsinki 2006, ISBN 952-10-2122-5

103. The ALICE Collaboration, K. Aamodt et al., The ALICE experiment at the CERN LHC, 2008 JINST 3 S08002

104. The ATLAS Collaboration, G. Aad et al., The ATLAS Experiment at the CERN Large Hadron Collider, 2008 JINST 3 S08003

105. The ATLAS TRT collaboration, E. Abat et al., The ATLAS TRT Barrel Detector, 2008 JINST $3 \mathrm{P} 02014$ 
106. T. Cornelissen and W. Liebig, ATLAS Inner Detector Results from the 2004 Combined Test Beam Data, Nuclear Physics B (Proc. Suppl.) 172 (2007) 292-295

Tuan Vu Anh, Private communication, February 2009

107. ALICE collaboration, ALICE transition-radiation detector: Technical Design Report, CERNLHCC-2001-021

108. C. Adler et al., Electron/pion identification with ALICE TRD prototypes using a neural network algorithm, Nucl. Instr. and Meth. Phys. Res. A 552 (2005) 364-371

109. M. Holder and H. Suhr, Separation of electrons and pions by a transition radiation detector using flash ADCs, Nucl. Instr. and Meth. Phys. Res. A 263 (1988) 319

110. A. Andronic, Electron identification performance with ALICE TRD prototypes, Nucl. Instr. and Meth. Phys. Res. A 522 (2004) 40-44

111. ALICE Collaboration, S. Acharya et al., The ALICE Transition Radiation Detector: construction, operation, and performance, arXiv:1709.02743v1 [physics.ins-det] 8 Sep 2017, CERN-EP-2017-222 29 August 2017

Open Access This chapter is licensed under the terms of the Creative Commons Attribution 4.0 International License (http://creativecommons.org/licenses/by/4.0/), which permits use, sharing, adaptation, distribution and reproduction in any medium or format, as long as you give appropriate credit to the original author(s) and the source, provide a link to the Creative Commons licence and indicate if changes were made.

The images or other third party material in this chapter are included in the chapter's Creative Commons licence, unless indicated otherwise in a credit line to the material. If material is not included in the chapter's Creative Commons licence and your intended use is not permitted by statutory regulation or exceeds the permitted use, you will need to obtain permission directly from the copyright holder. 